\title{
Inflammation and the Brain Disorders: A Review
}

\section{Rahimeh Bargi ${ }^{1}$, Hossein Salmani²*, Fereshteh Asgharzadeh Yazdi², Mahmoud Hosseini ${ }^{1}$}

${ }^{1}$ Neurocognitive Research Center, School of Medicine, Mashhad University of Medical Sciences, Mashhad, Iran ${ }^{2}$ Neurogenic Inflammation Research Center, School of Medicine, Mashhad University of Medical Sciences, Mashhad, Iran

$\begin{array}{llr} & \text { Article Info: } & \\ \text { Received: } 10 \text { May } 2016 & \text { Revised: } 28 \text { Jun } 2016 & \text { Accepted: } 18 \text { Sep } 2016\end{array}$

\section{ABSTRACT}

Introduction: Inflammation is a physiological response of immune system against internal and external harmful stimulus. However, inflammation is a double-edged sword and can be harmful if it is not properly controlled. Nowadays inflammation is considered as an important factor in the pathophysiology of neurological diseases. Neuroinflammation can be as a result of direct damage to the brain tissues or induced by systemic inflammation. This process is characterized by microglial activation, stimulates astrocytes, blood - brain barrier breakdown and consequent increase in the permeability, penetration of peripheral immune cells into parenchyma of the central nervous system, excessive production of cytokines, nitric oxide, reactive oxygen species as well as prostaglandins and finally, neuronal damage and death. In this study, neuroinflammation and its role in the pathophysiology of brain diseases, including Alzheimer $>$ s disease, Parkinson $>$ s disease, Huntington $>$ s disease, depression, anxiety, epilepsy, schizophrenia and autism are outlined and discussed. Conclusion: Although inflammation has a prominent role in the pathogenesis of neurological diseases, there are no effective and safe treatments to control deregulated inflammatory processes in the brain. The medications usually used to control inflammation are not fully effective in the brain due to lack of BBB penetration. Since many inflammatory processes in the brain is beneficial, modulation of inflammatory responses will be a more efficient therapeutic approach than suppression of inflammatory processes.

\section{Key words:}

1. Neurodegenerative Diseases

2. Microglia

3. Nervous System Diseases

4. Inflammation

*Corresponding Author: Hossein Salmani

E-mail: SalmaniH921@mums.ac.ir 


\section{التهاب و بيمارىهاى مغزى: يك مطالعةٌ مرورى}

\section{رحيمه بركَى'، حسين سلمانى זّ، فرشته اصغر زاده يزدى'، محمود حسينى'}

'مركز تحقيقات علوم شناختى، دانشكده يزشكى، دانشگاه علوم يزشكى مشهد، مشهر، ايران

'مركز تحقيقات التهاب نوروزنيك، دانشكده يزشكى، دانشكاه علوم يزشكى مشهر، مشهد، ايران

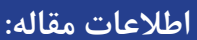

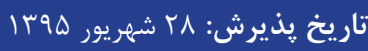

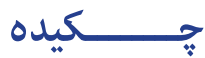

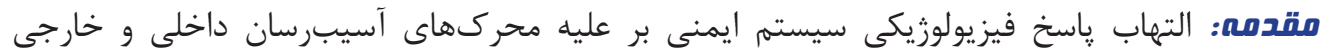

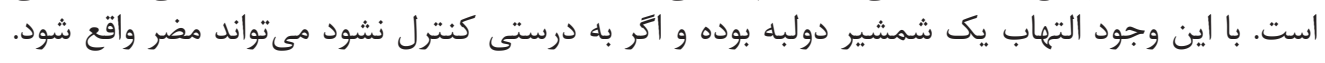

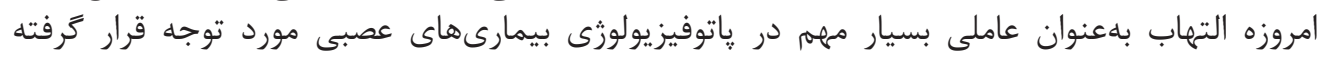

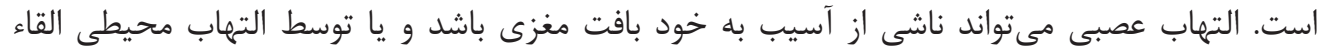

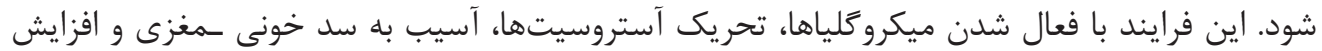

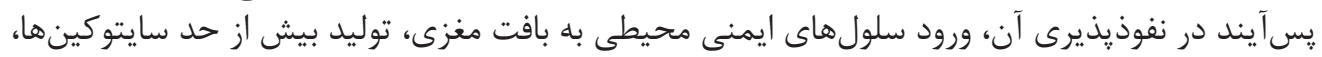

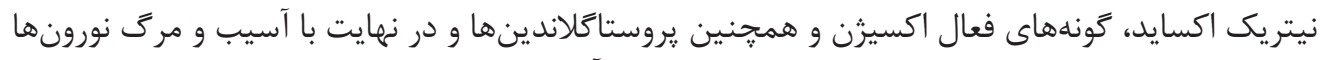

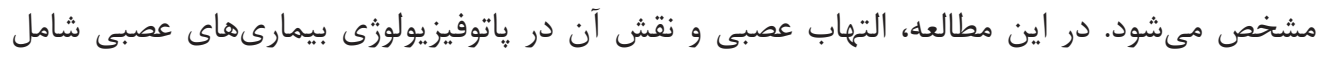

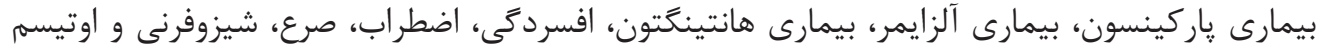

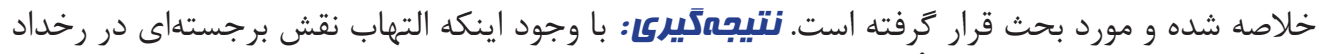

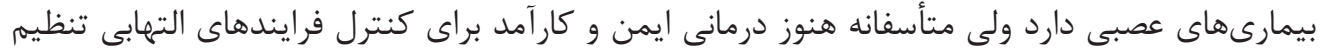

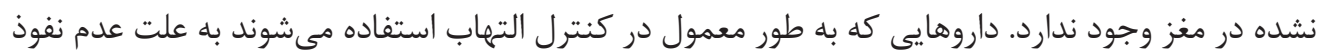

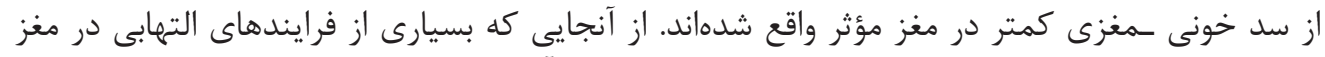

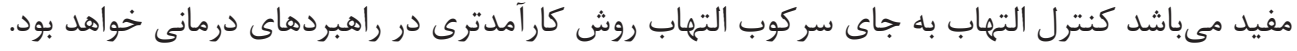

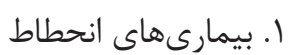

نورونى

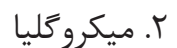

ז. بيمارىهاى سيستم ميكم

عصبى

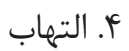

" نويسنده مسئول: حسين سلمانى

آدرس الكترونيكى: SalmaniH921@mums.ac.ir 


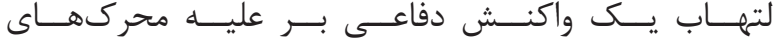

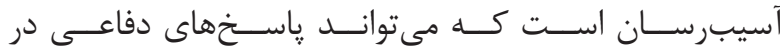

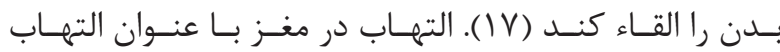

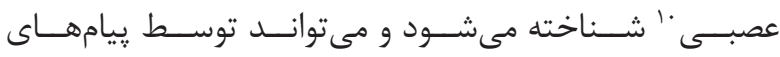

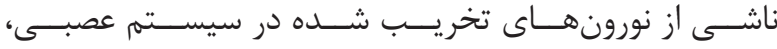

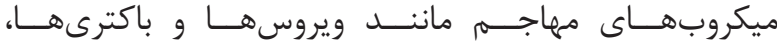

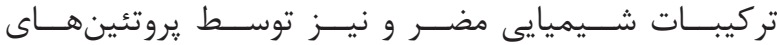

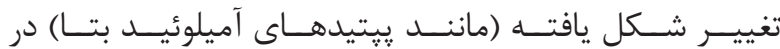

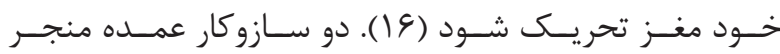

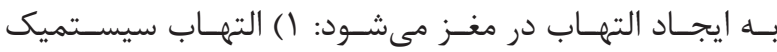

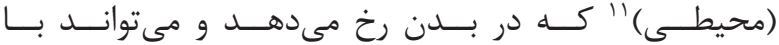

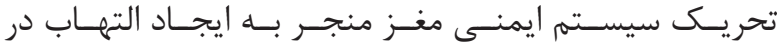

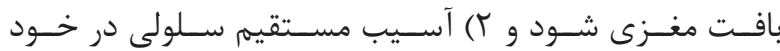

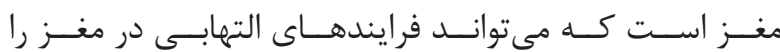

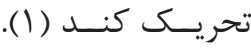

التهـاب عصبـى در شـرايط پاتولـوزى متعـدادى جـون سـكته،

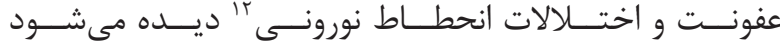

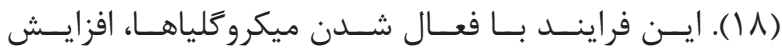

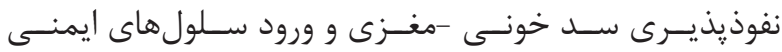

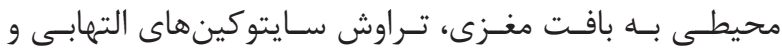

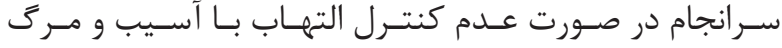

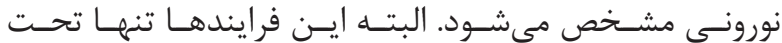

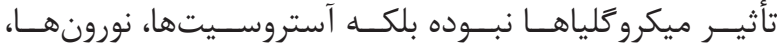

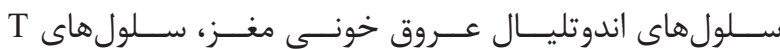

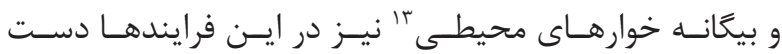

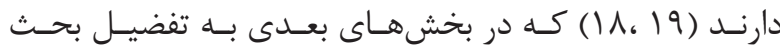

خواهنــد شـد (رند

\section{1-1 - نقش ميكروگلياها در التهاب عصبى}

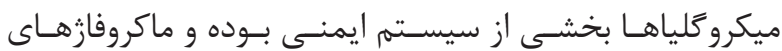

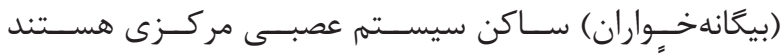

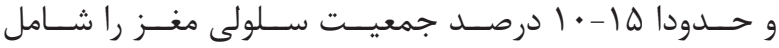

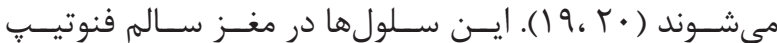

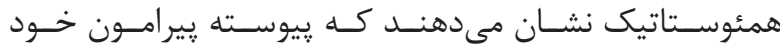

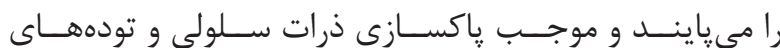

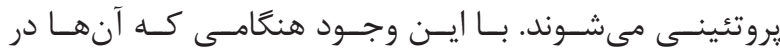

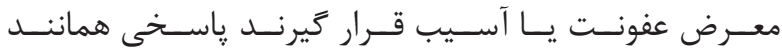

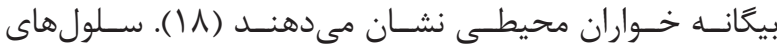

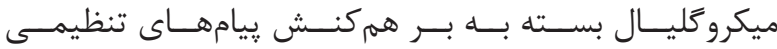

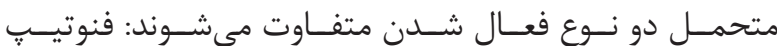

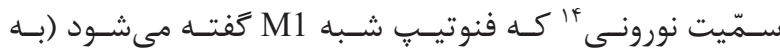

\footnotetext{
${ }^{1}$ Hypertension

${ }^{2}$ Alzheimer's disease

${ }^{3}$ Parkinson's disease

${ }^{4}$ Bipolar disorder

${ }^{5}$ Schizophrenia

${ }^{6}$ Depression

${ }^{7}$ Epilepsy
}

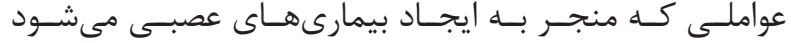

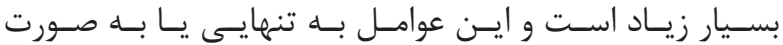

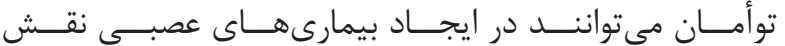

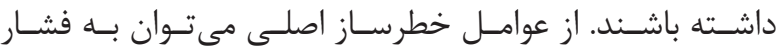

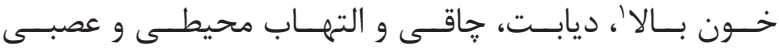

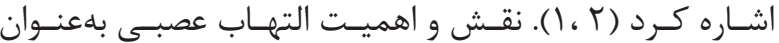

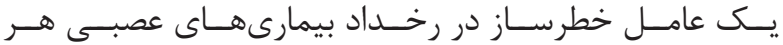

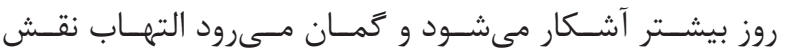

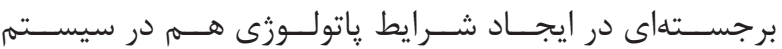

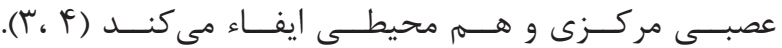

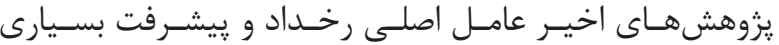

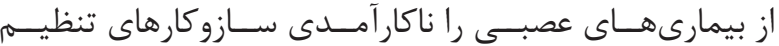

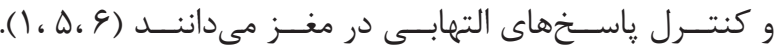

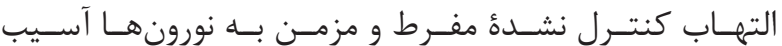

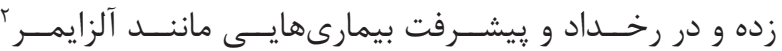

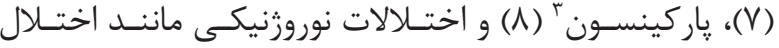

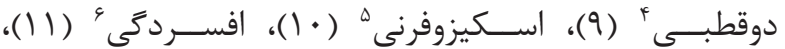

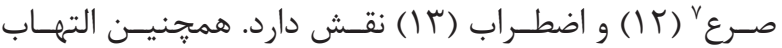

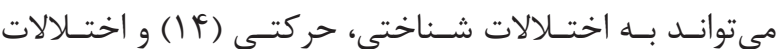

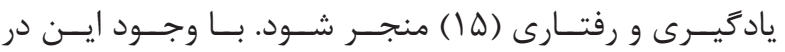

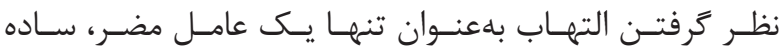

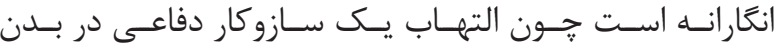

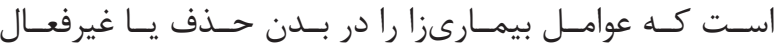

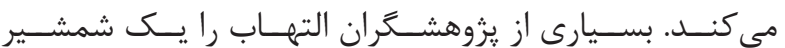

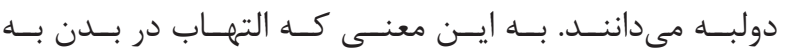

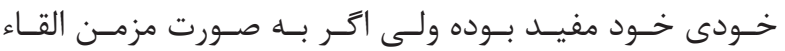

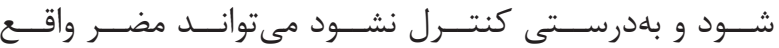

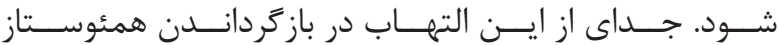

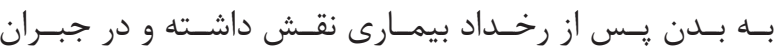

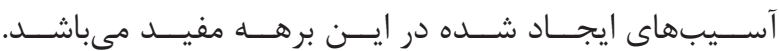

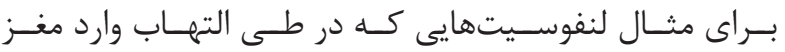

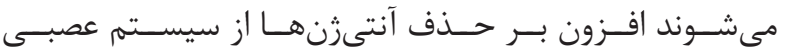

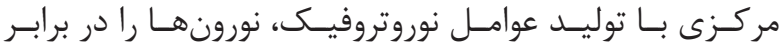

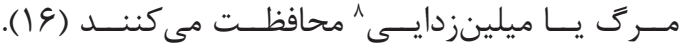

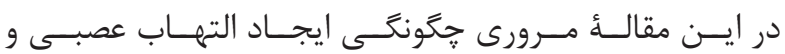

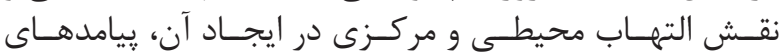

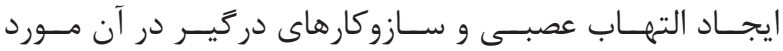

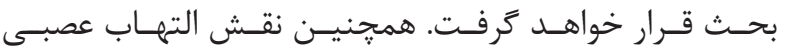

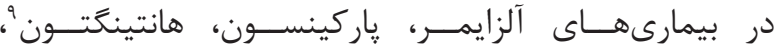

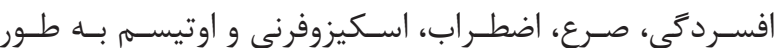

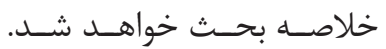

\footnotetext{
${ }^{8}$ Demyelination

${ }^{9}$ Huntington's disease

${ }^{10}$ Neuroinflammation

${ }^{11}$ Peripheral inflammation (systemic inflammation)

${ }^{12}$ Neurodegenerative disorders

${ }^{13}$ Peripheral macrophages

${ }^{14}$ Neurotoxic phenotype
} 


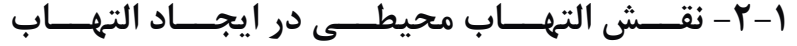

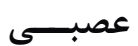

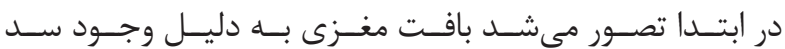

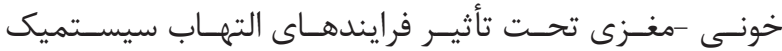

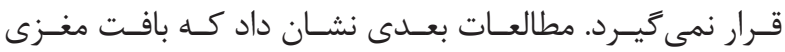

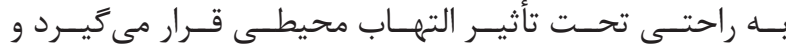

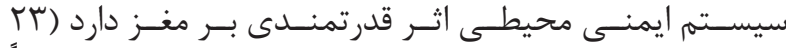

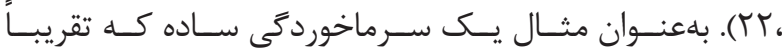

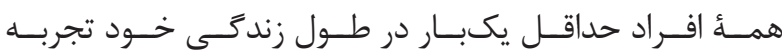

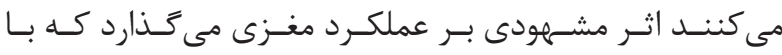

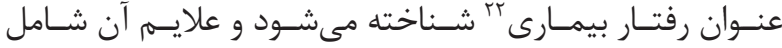

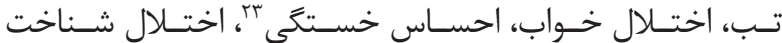

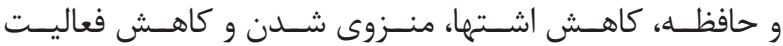

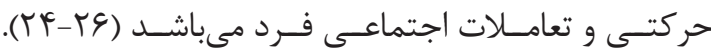

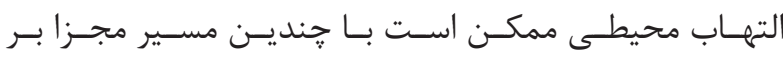

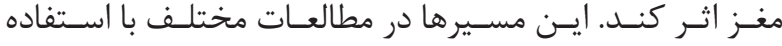

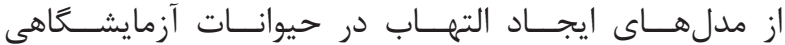

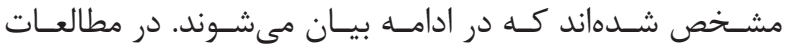

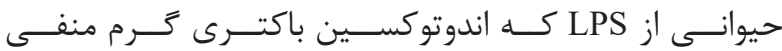

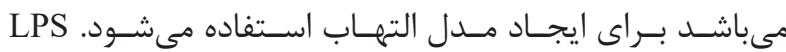

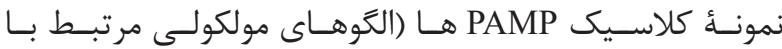

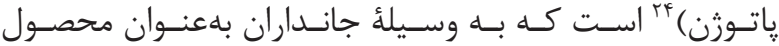

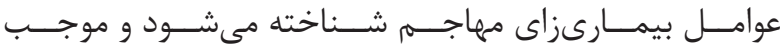

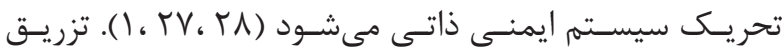

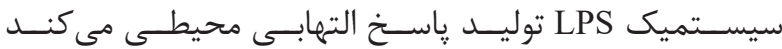

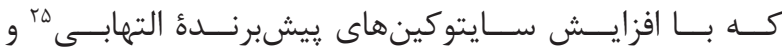

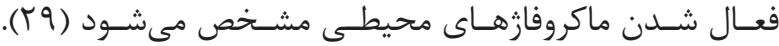

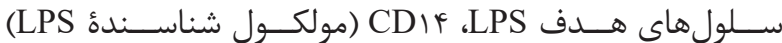

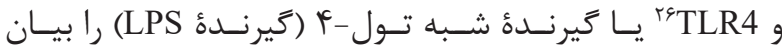

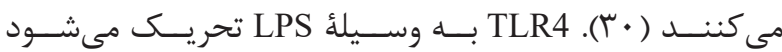

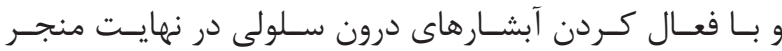

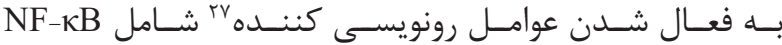

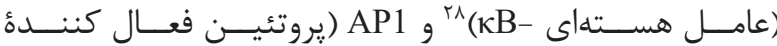

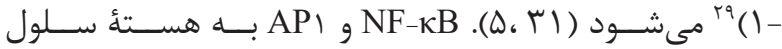

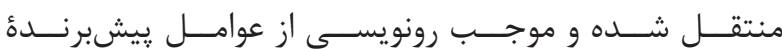

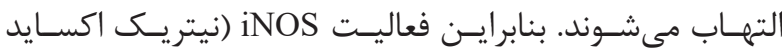

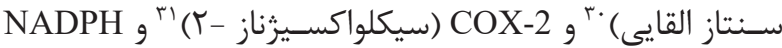

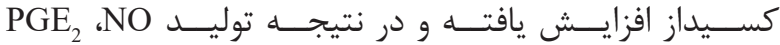

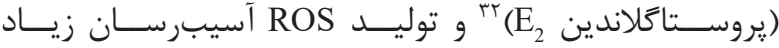

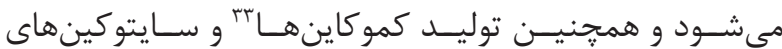

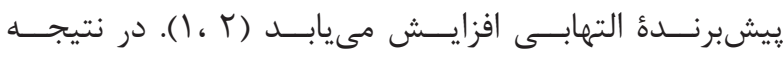

${ }^{15}$ Reactive oxygen species

${ }^{16}$ Neuroprotective phenotype

${ }^{17}$ Bacterial lipopolysaccharides

${ }^{18}$ Interferon- $\gamma$

${ }^{19}$ Tumor necrosis factor alpha

${ }^{20}$ Interleukin- $1 \beta$

${ }^{21}$ CC-chemokine ligand 2

${ }^{22}$ Sickness behavior

${ }^{23}$ Fatigue

${ }^{24}$ Pathogen associated molecular patterns

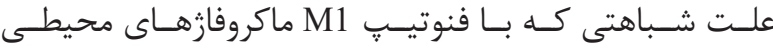

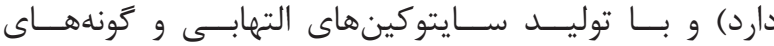

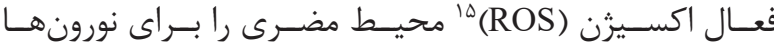

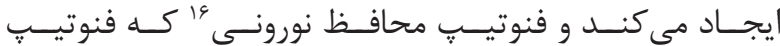

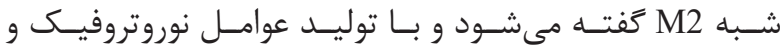

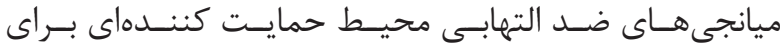

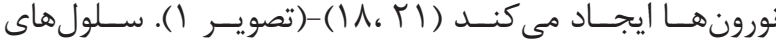

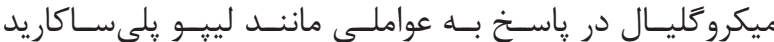

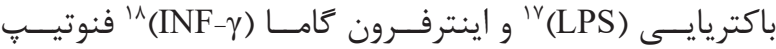

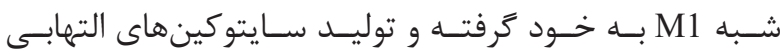

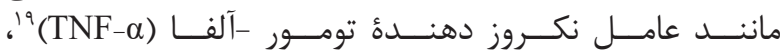

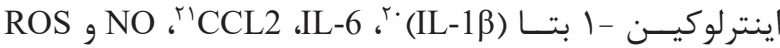

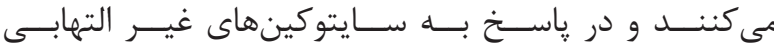

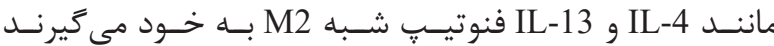

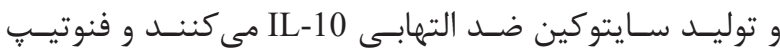

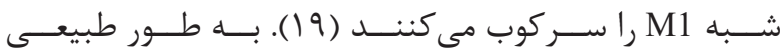

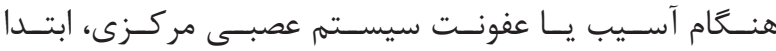

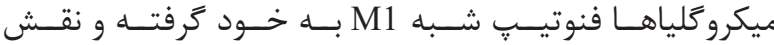

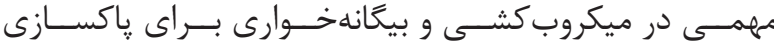

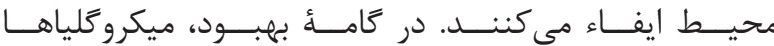

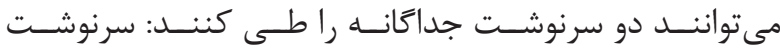

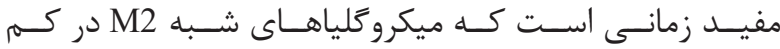

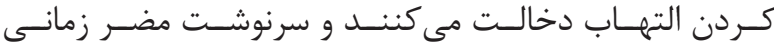

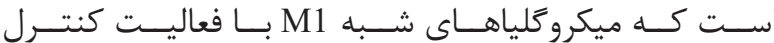

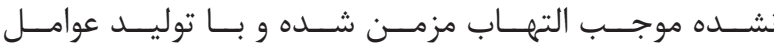

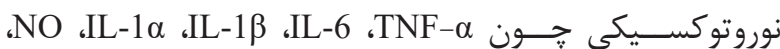

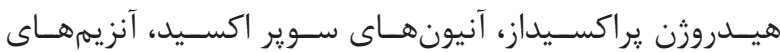

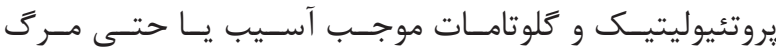

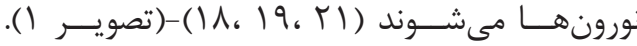

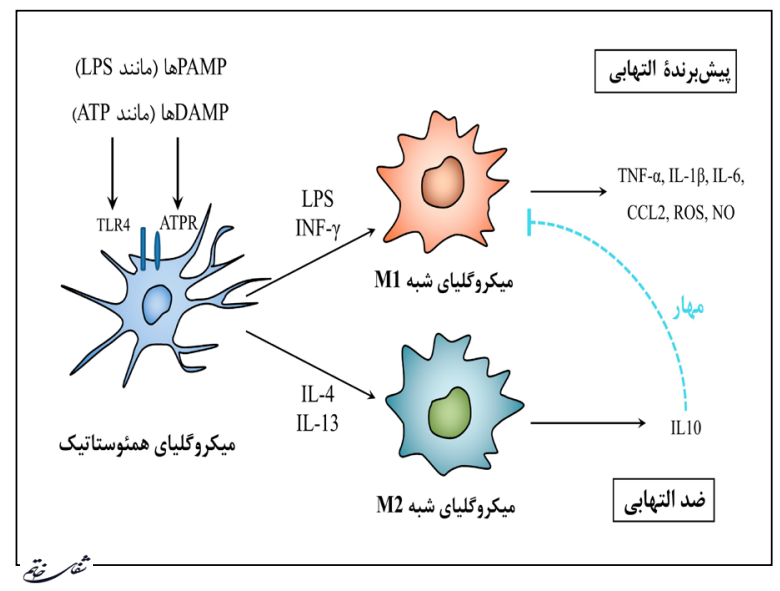

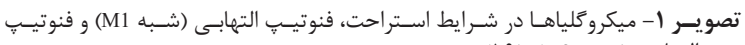

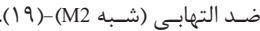

${ }^{25}$ Pro-inflammatory cytokines

26 Toll-like receptor-4

27 Transcription factors

${ }^{28}$ Nuclear factor- $\kappa \mathrm{B}$

${ }^{29}$ Activator protein-1

${ }^{30}$ Inducible nitric oxide synthase

${ }^{31}$ Cyclooxygenase -2

${ }^{32}$ Prostaglandin E2

${ }^{33}$ Chemokines 


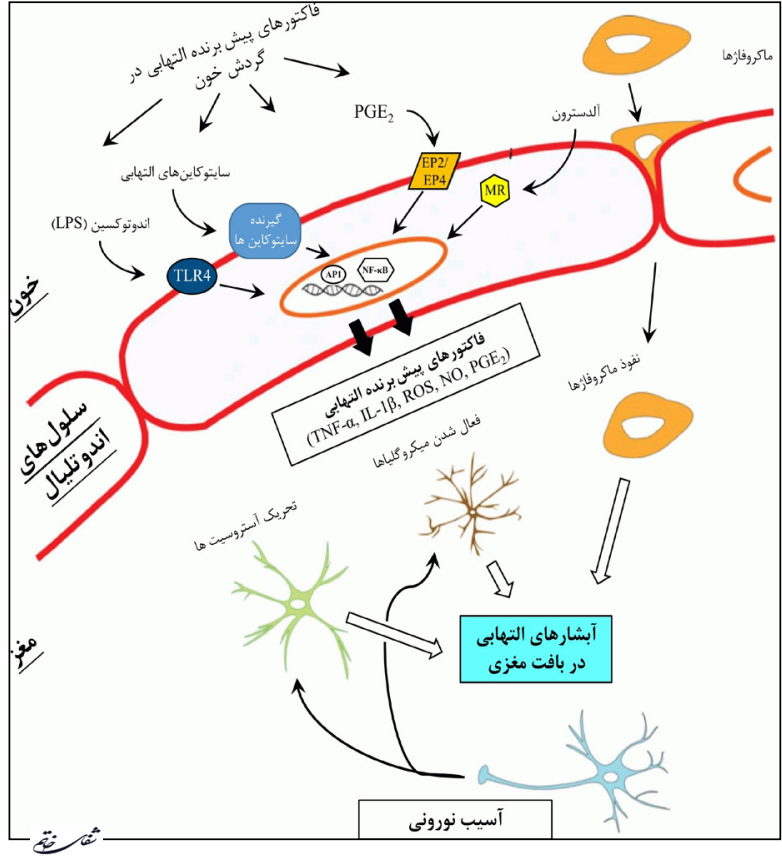

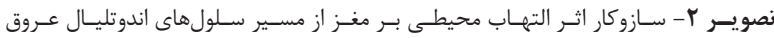

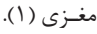

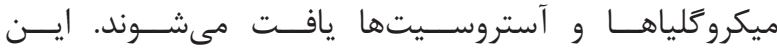

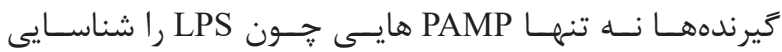

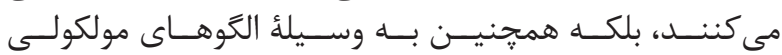

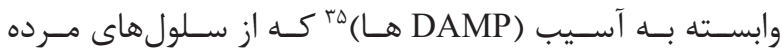

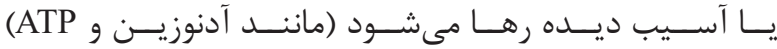

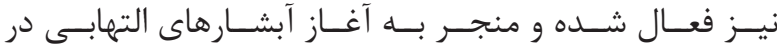

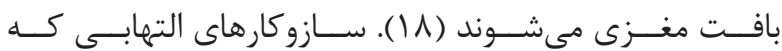

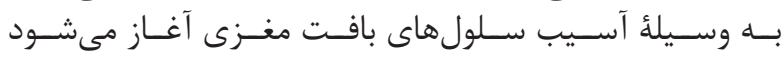

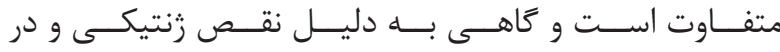

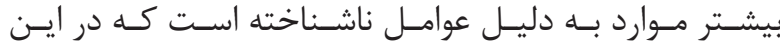

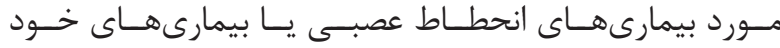

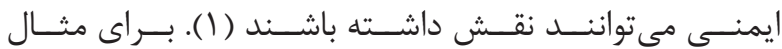

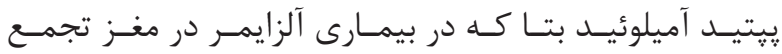

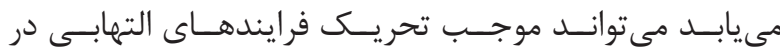

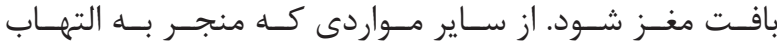

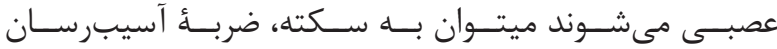

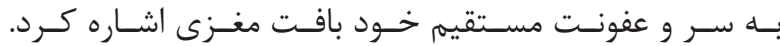
r- نقش التهاب در ايجاد بيمارىهاى عصبى r-1- التهاب و بيمارى آلزايمر

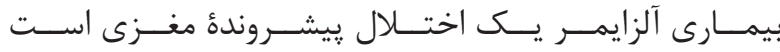

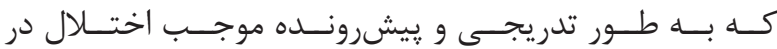

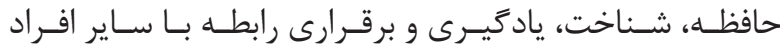

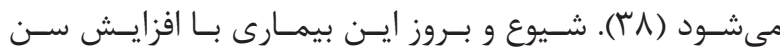

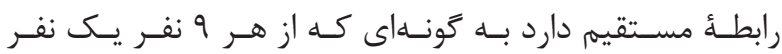

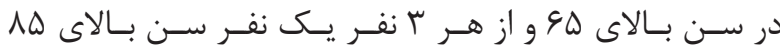

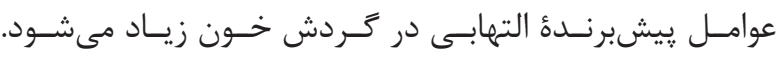

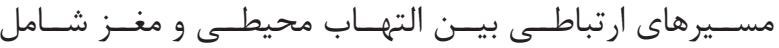

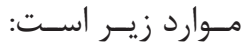

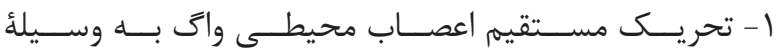

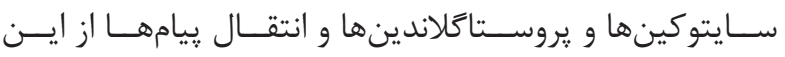

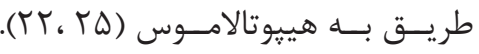

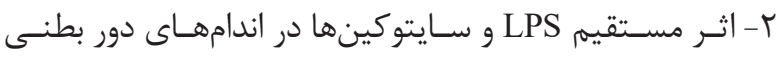

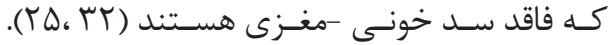

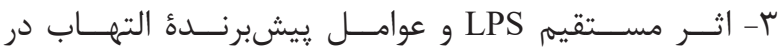

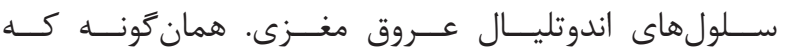

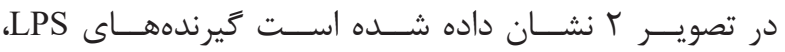

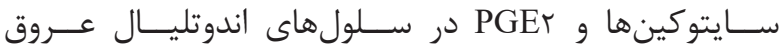

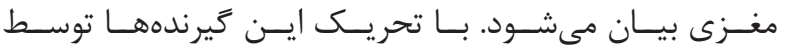

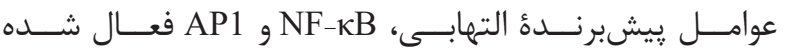

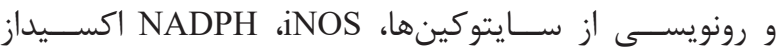

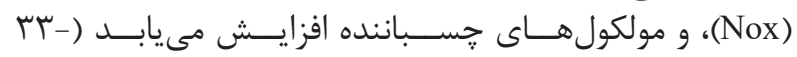

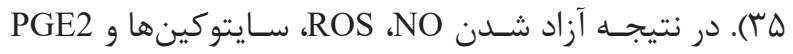

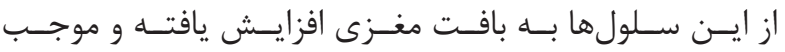

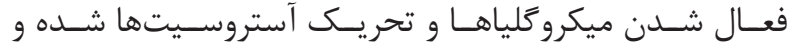

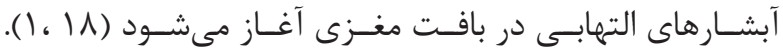

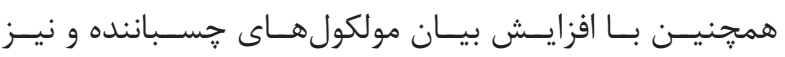

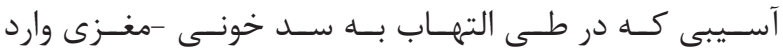

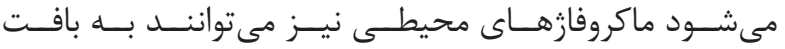

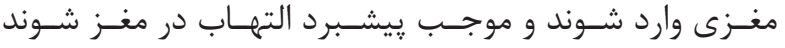

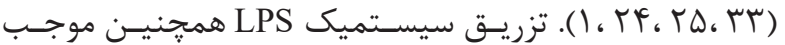

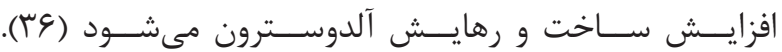

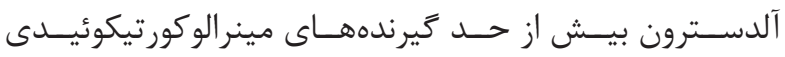

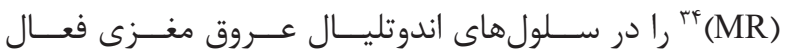

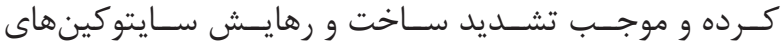

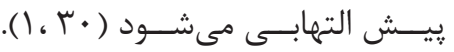

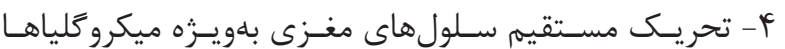

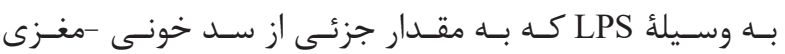

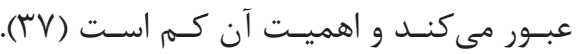

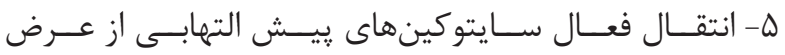

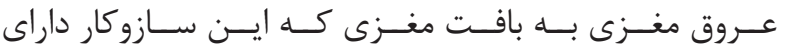

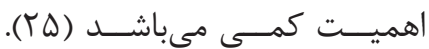

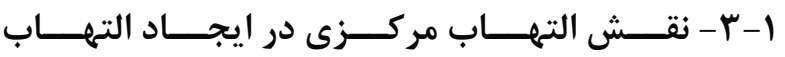

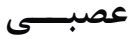

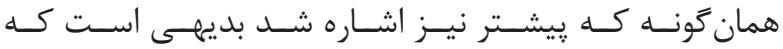

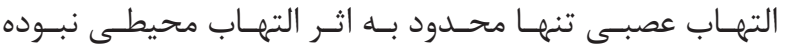

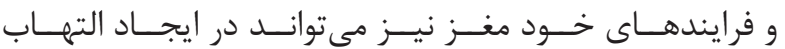

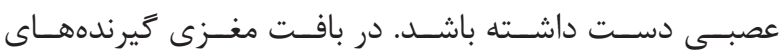

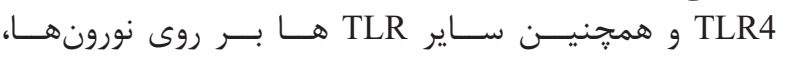

${ }^{34}$ Mineralocorticoid receptor

${ }^{35}$ Damage associative molecular pattern 
مهرمتريسـن بيمارىهــاى انحطــاط عصبــى محســوب مى شــود

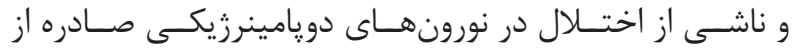

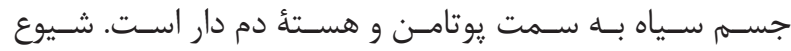

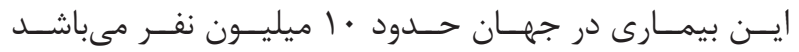

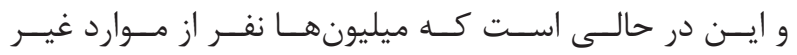

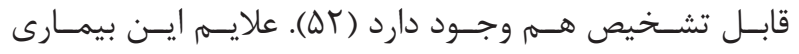

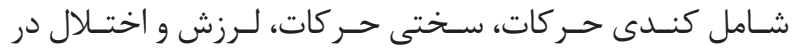

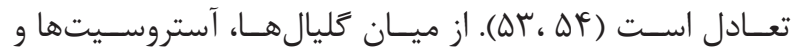

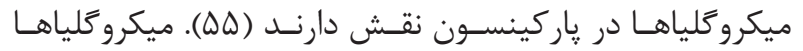

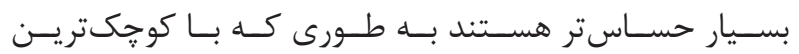

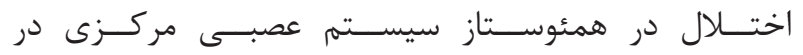

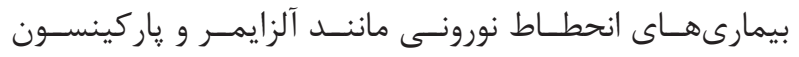

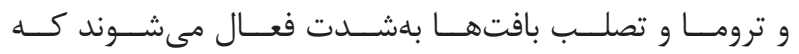

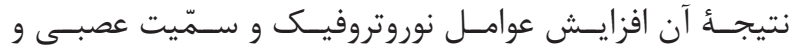

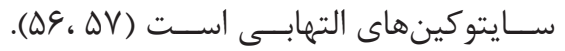

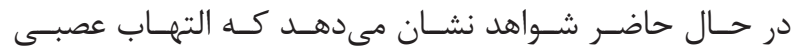

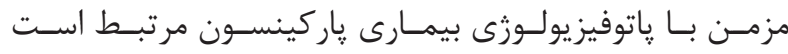

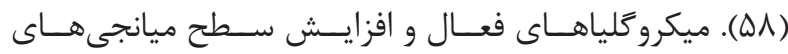

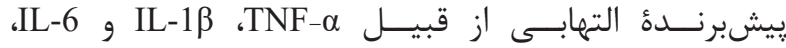

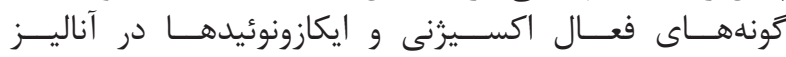

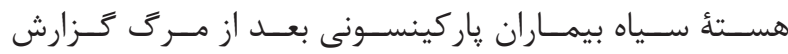

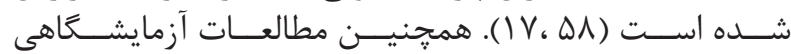

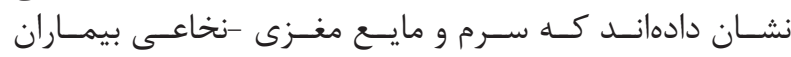

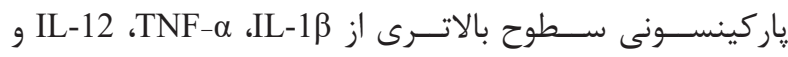
همجنيــن لنفوسـيتهاى

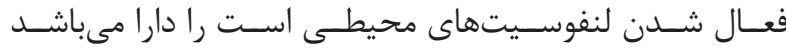

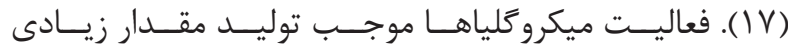

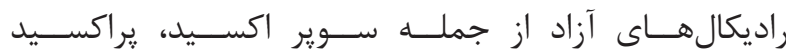

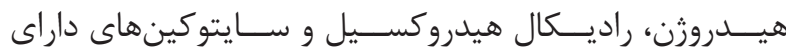
سـمّيت سـلولى از جملـه هN

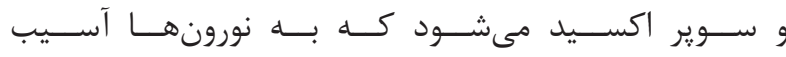

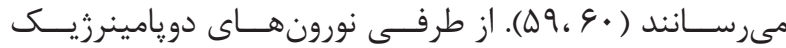

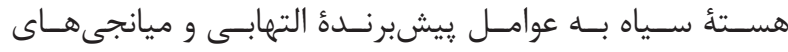

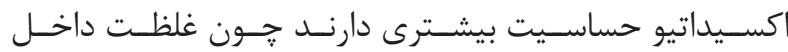

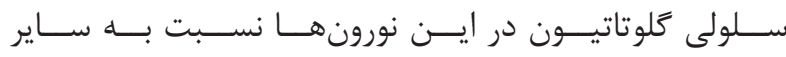

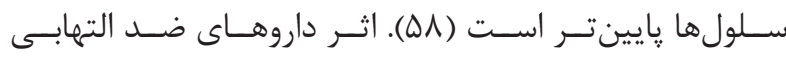

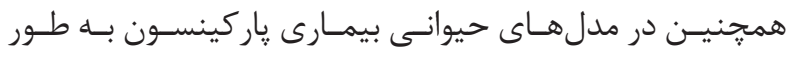

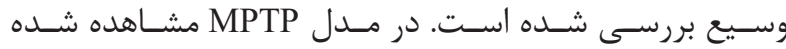

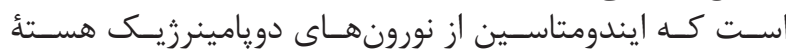

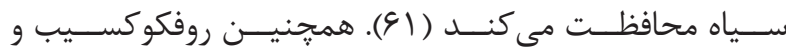

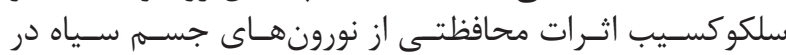

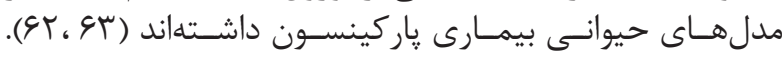

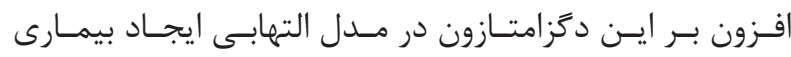

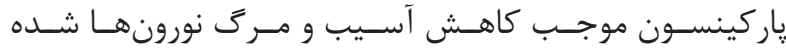

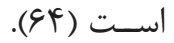

r-r- التهاب و بيمارى هانتينَتون

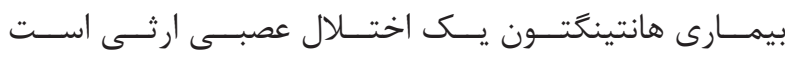

${ }^{36}$ Oxidative stress

${ }^{37} \mathrm{C}$ reactive protein

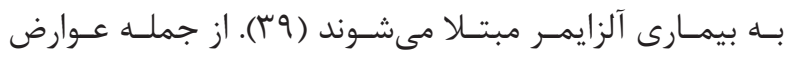

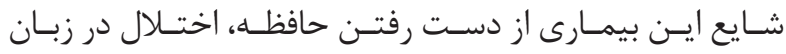

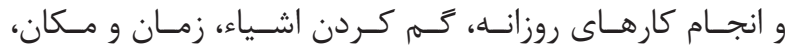

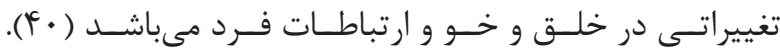

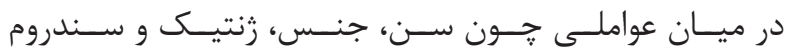

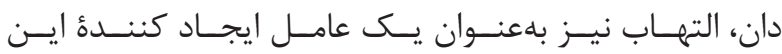

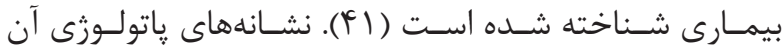

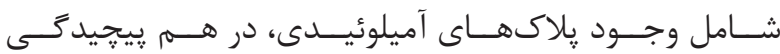

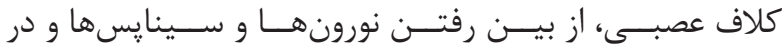

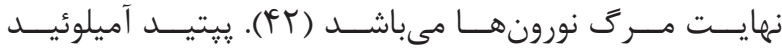

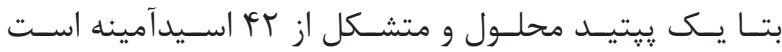

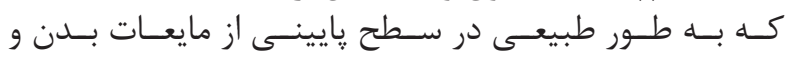

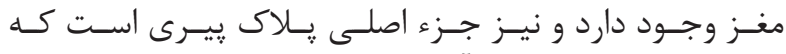

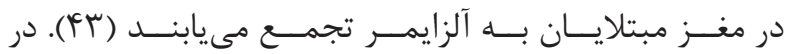

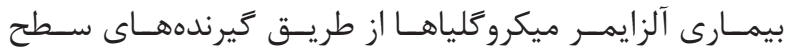

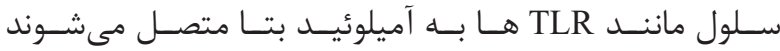

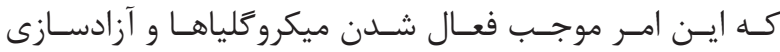

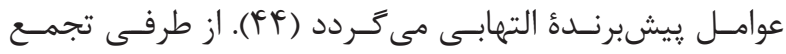

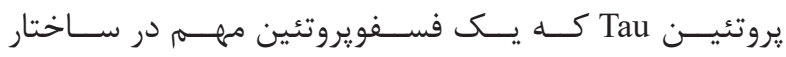

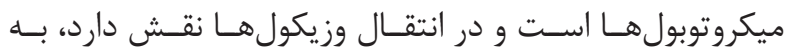

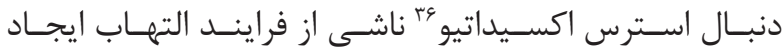

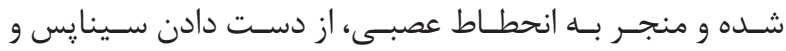

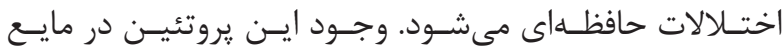

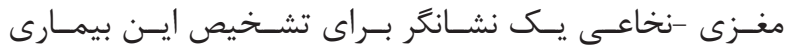

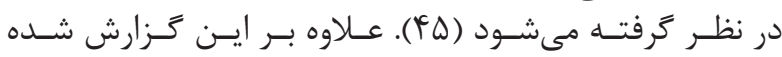

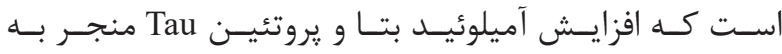

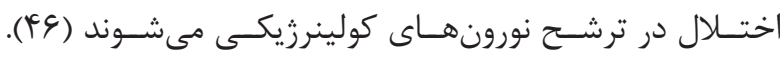

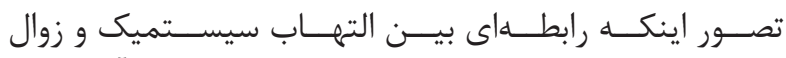

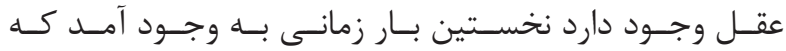

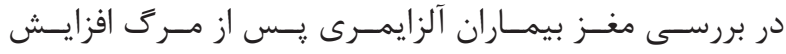

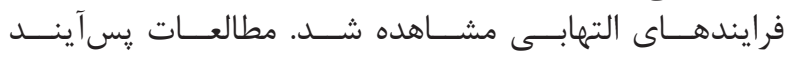

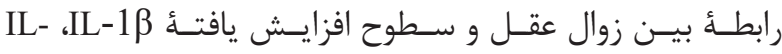

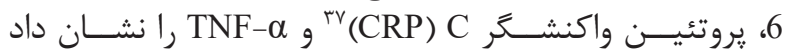

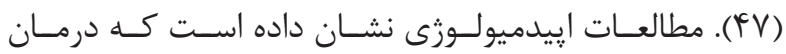

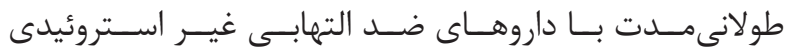

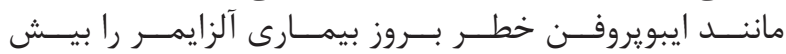

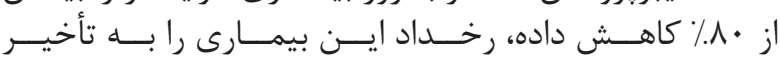

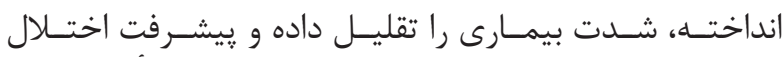

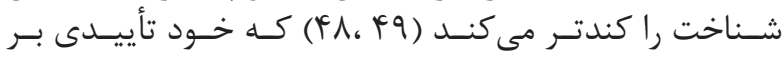

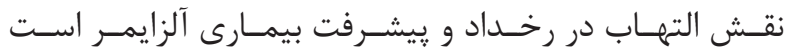

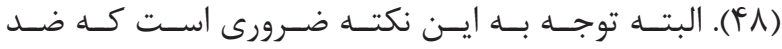

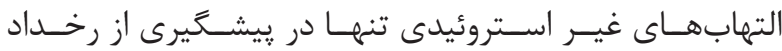

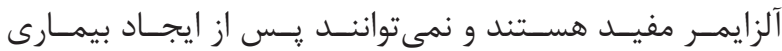

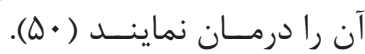

r-r - التهاب و بيمارى یاركينسون

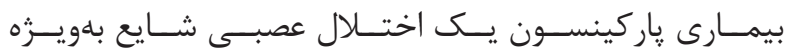

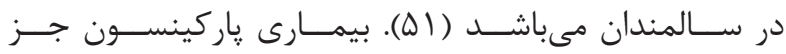




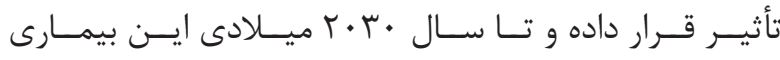

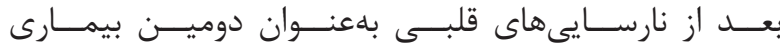

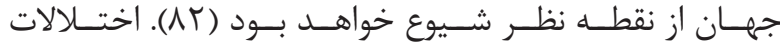

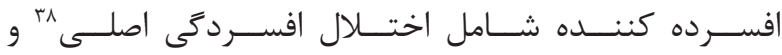

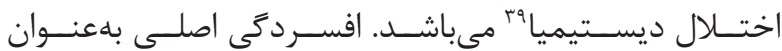

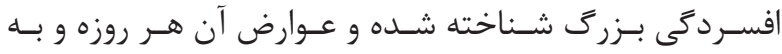

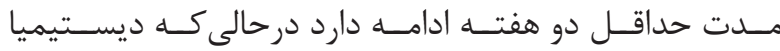

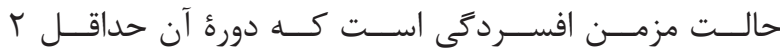

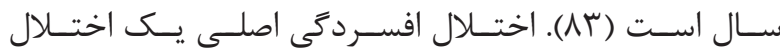

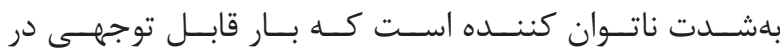

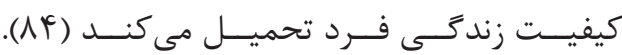

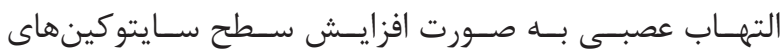

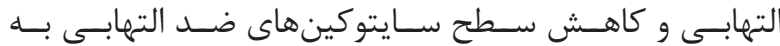

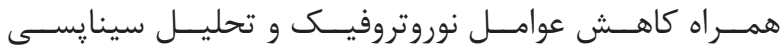

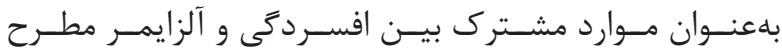

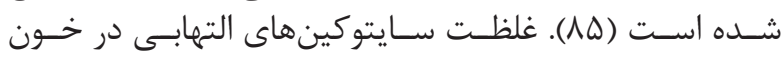

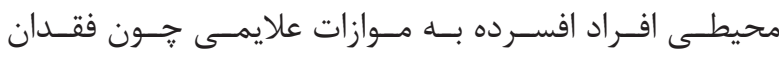

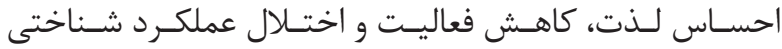

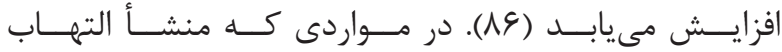

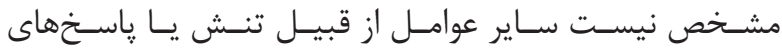

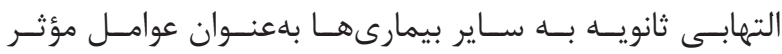

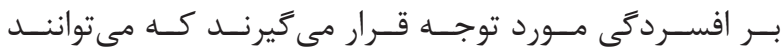

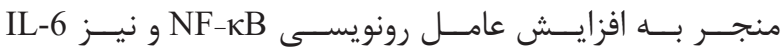

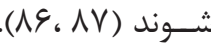

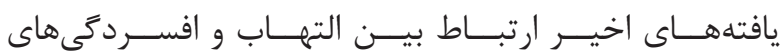

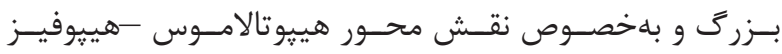

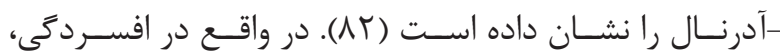

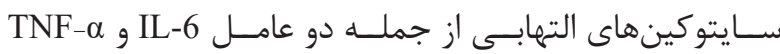

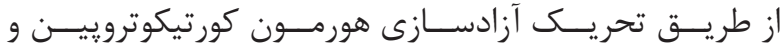

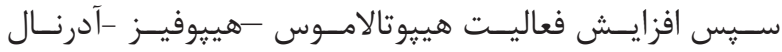

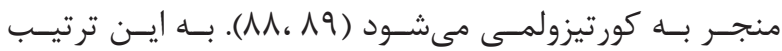

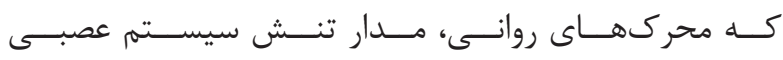

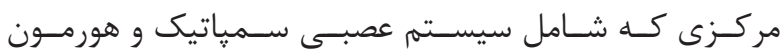

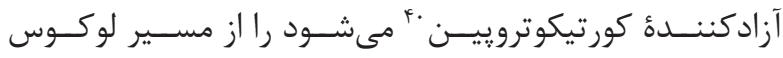

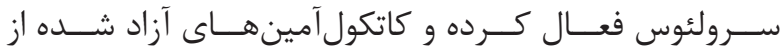

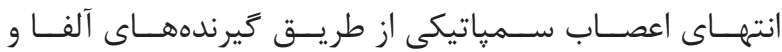

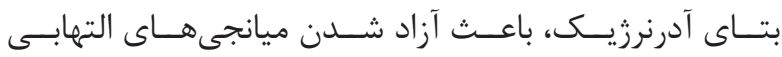

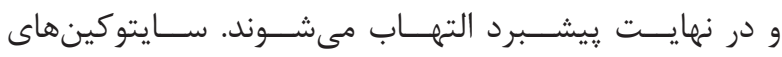

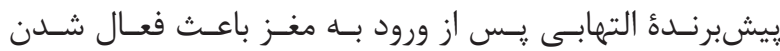

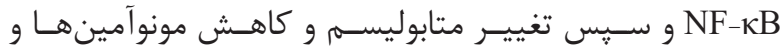

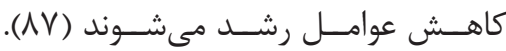

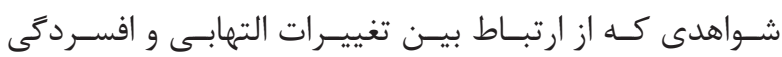

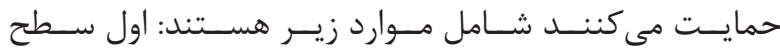

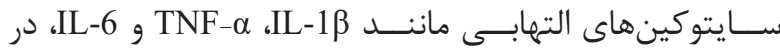

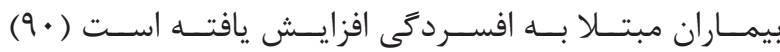

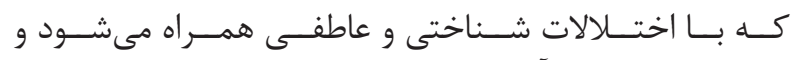

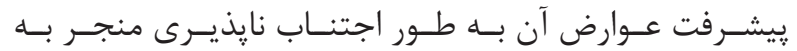

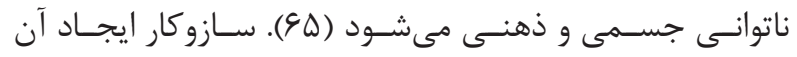

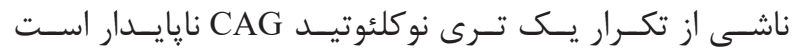

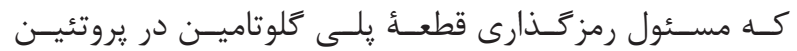

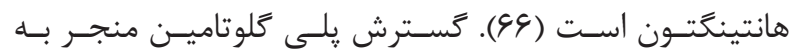

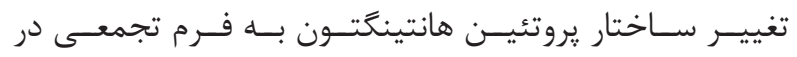

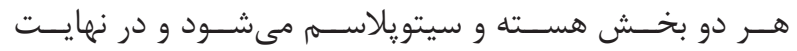

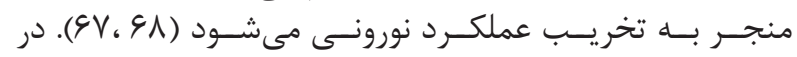

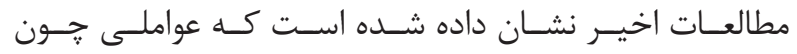

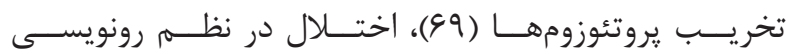

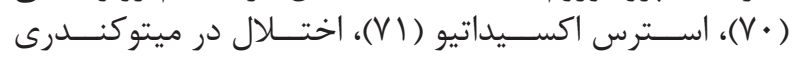

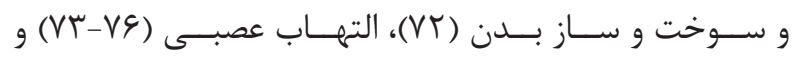

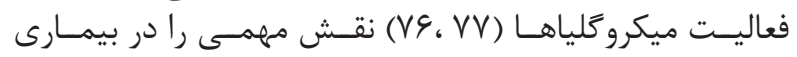

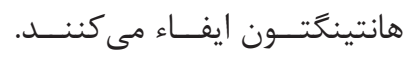

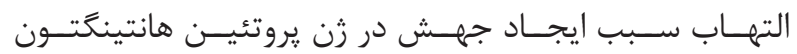

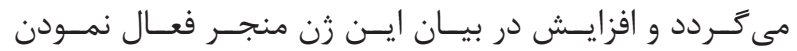

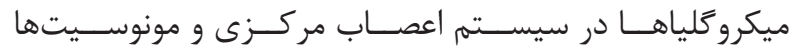

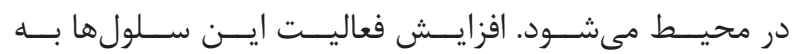

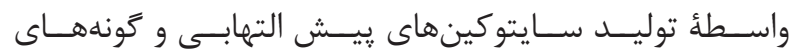

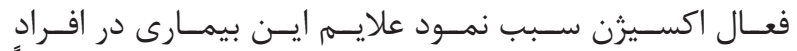

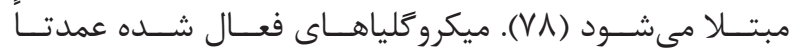

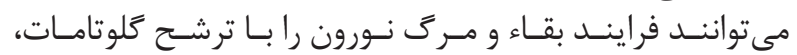

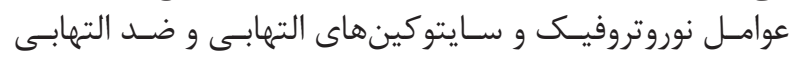

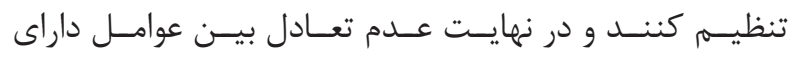

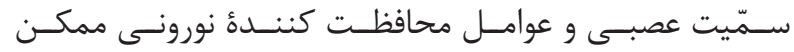

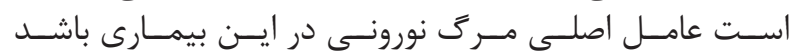

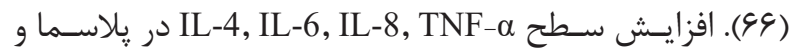

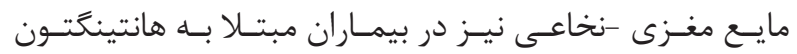

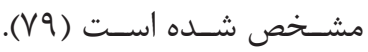

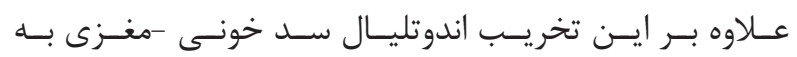

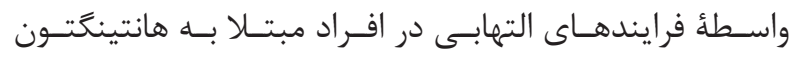

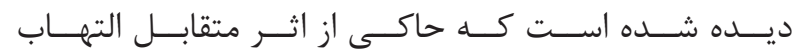

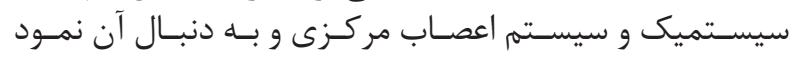

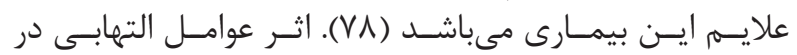

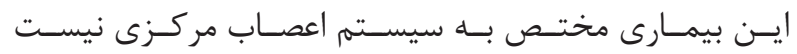

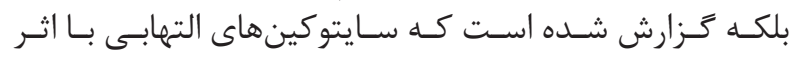

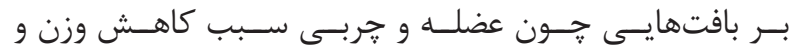

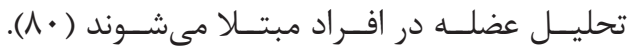

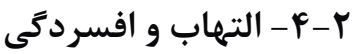

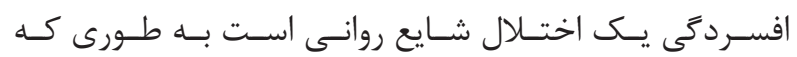

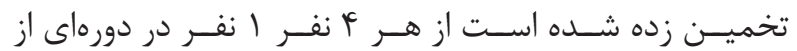

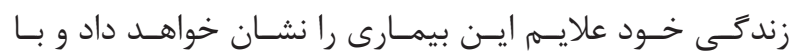

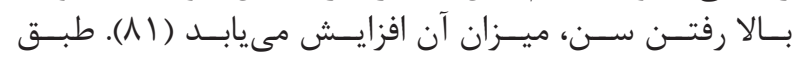

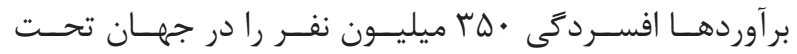

${ }^{38}$ Major depressive disorder

${ }^{39}$ Dysthymia

${ }^{40}$ Corticotropin-releasing hormone $(\mathrm{CRH})$ 


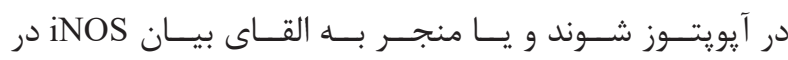

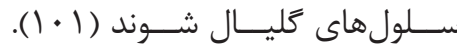

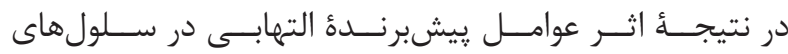

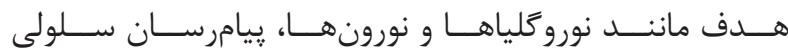

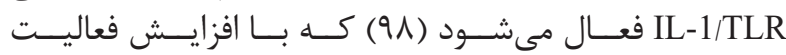

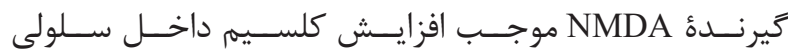

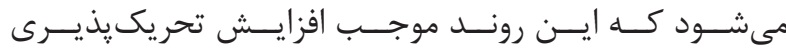

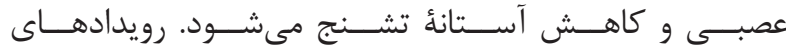

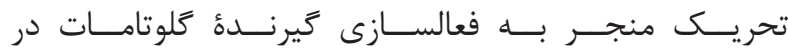

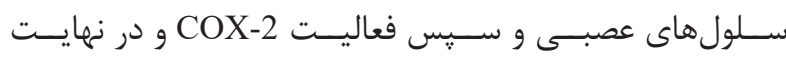

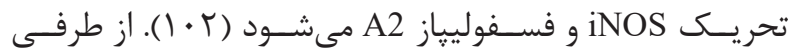

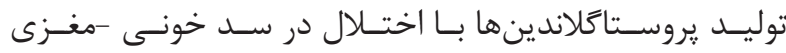

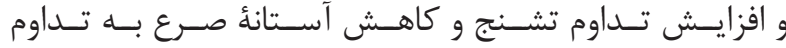

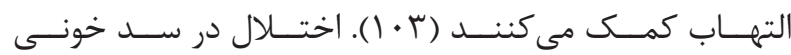

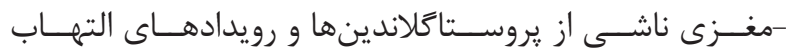

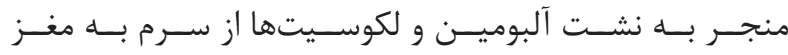

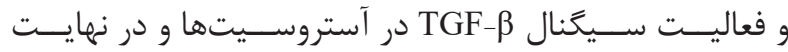

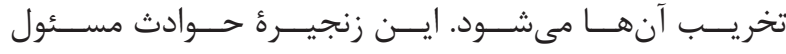

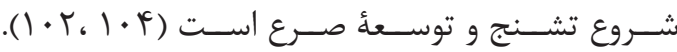

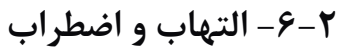

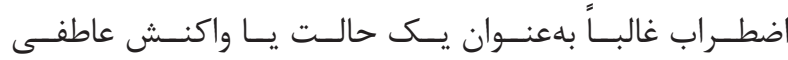

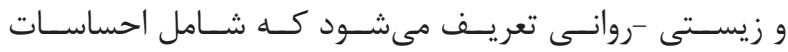

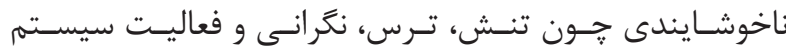

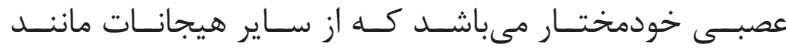

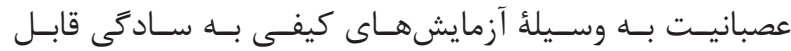

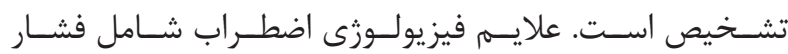

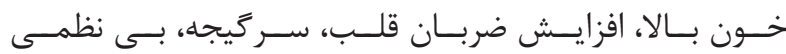

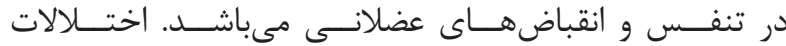

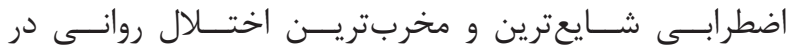

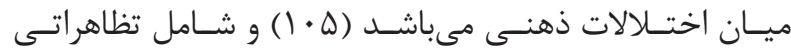

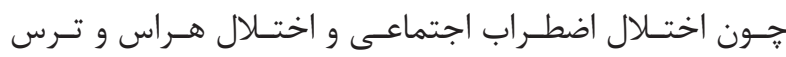

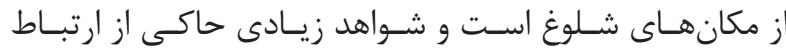

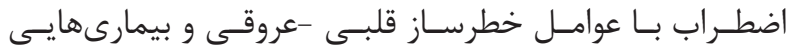

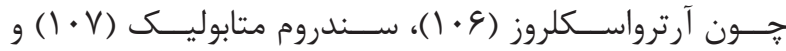

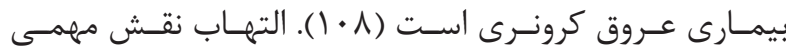

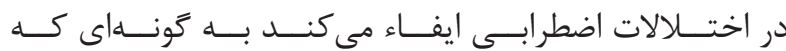

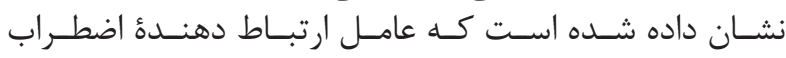

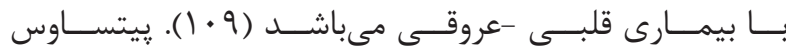

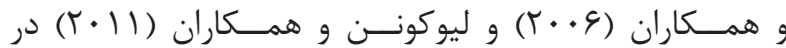

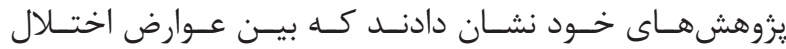

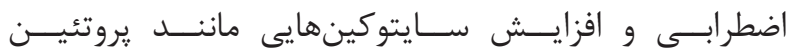

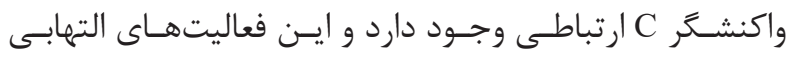

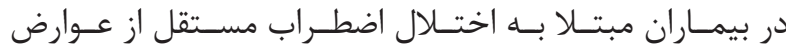

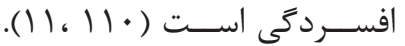

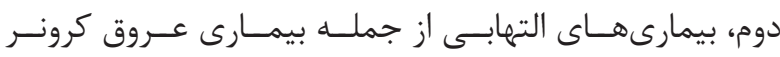

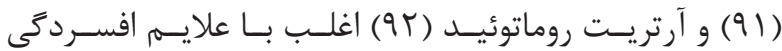

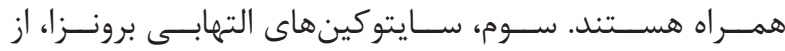

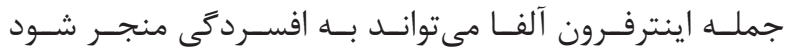

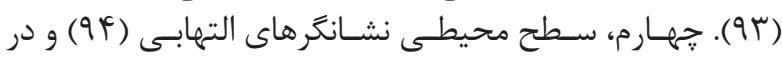

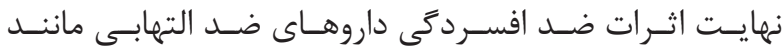

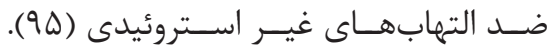

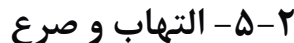

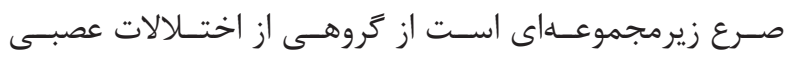

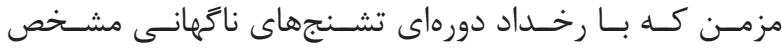

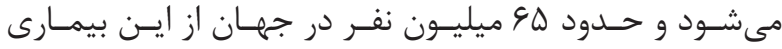

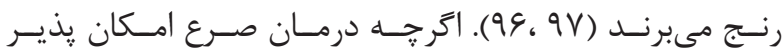

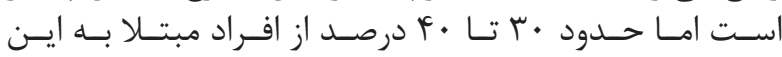

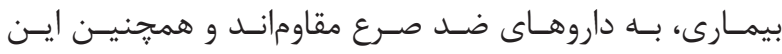

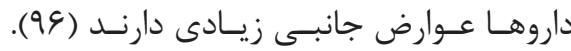

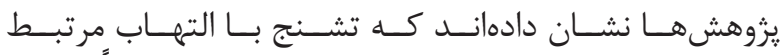

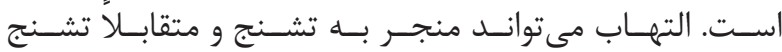

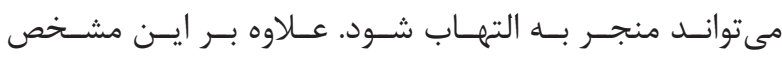

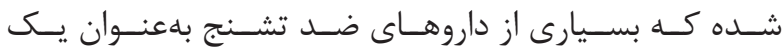

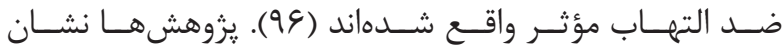

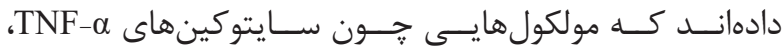
IL-1

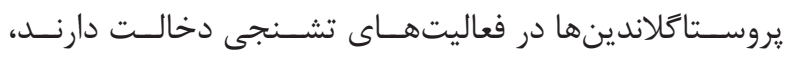

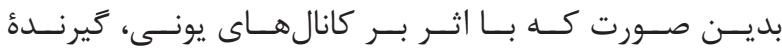

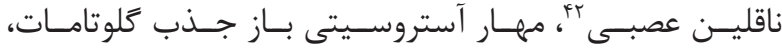

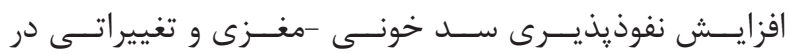

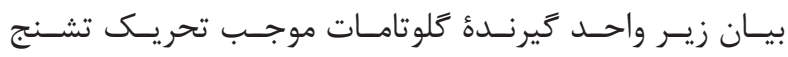

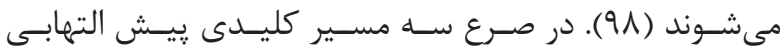

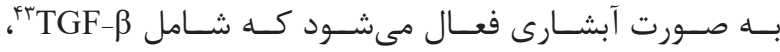

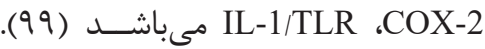

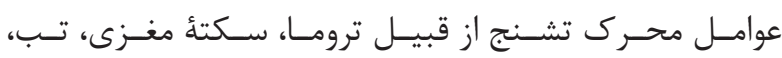

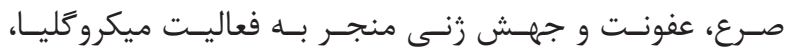

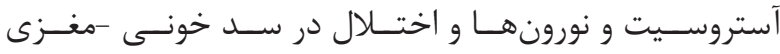

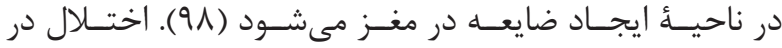

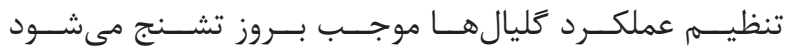

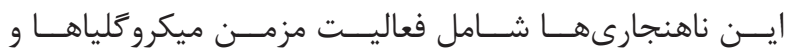

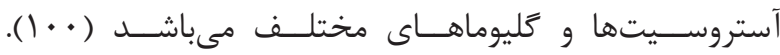

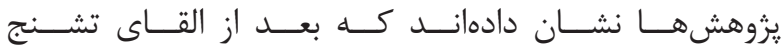

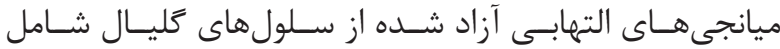

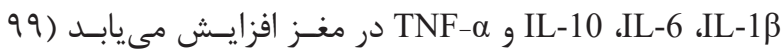

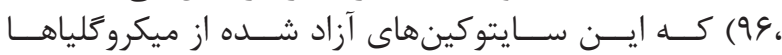

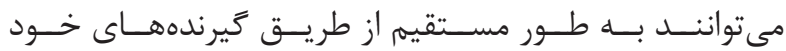

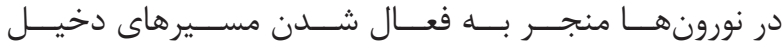

${ }^{41}$ High mobility group box 1

${ }^{42}$ Neurotransmitters

${ }^{43}$ Transforming growth factor $\beta$ 


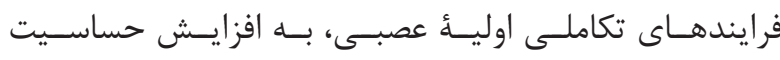

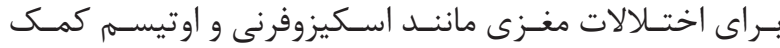

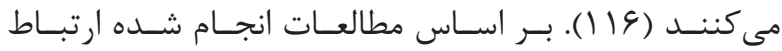

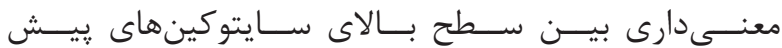

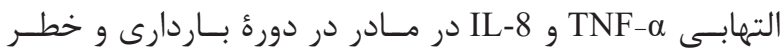

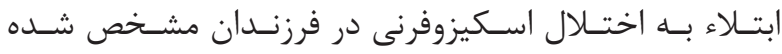

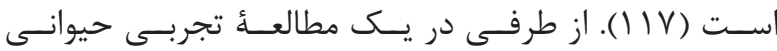

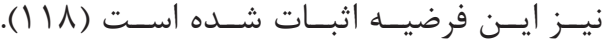

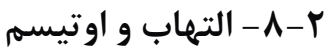

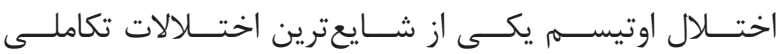

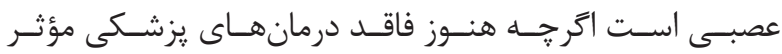

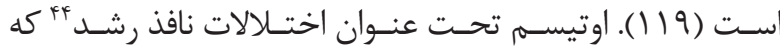

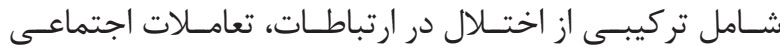

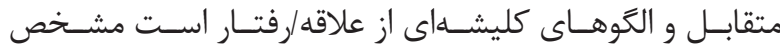

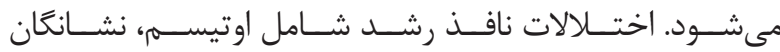

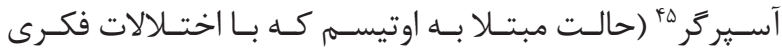

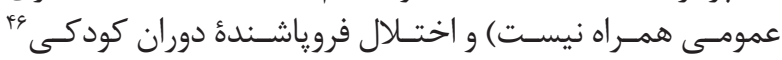

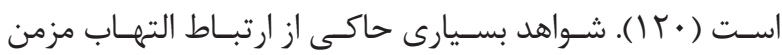

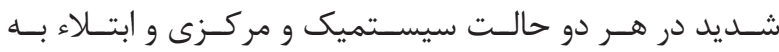

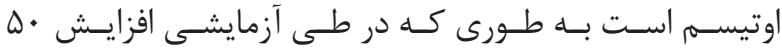

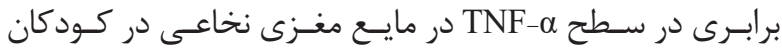

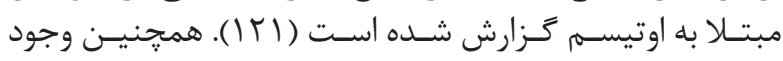

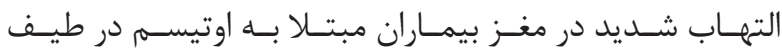

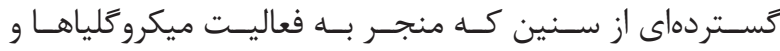

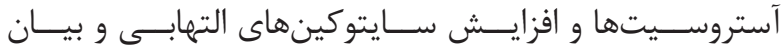

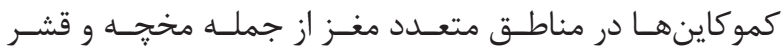

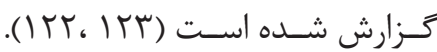

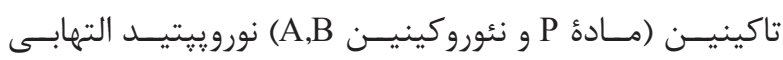

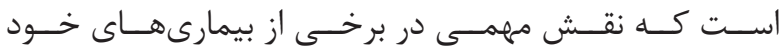

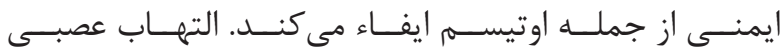

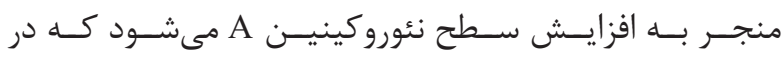

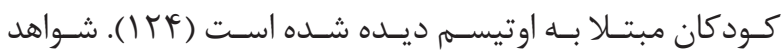

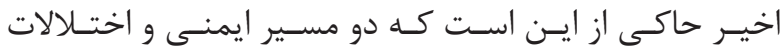

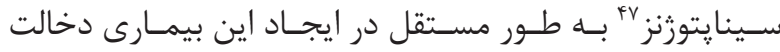

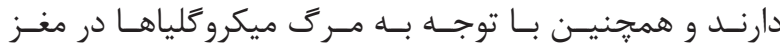

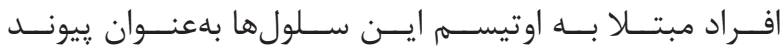

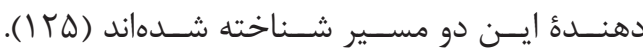

نتيجه

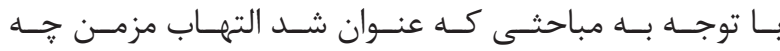

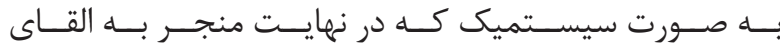

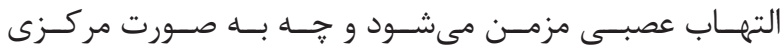

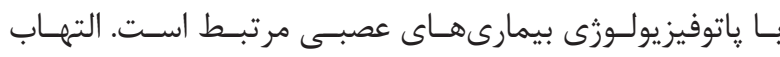

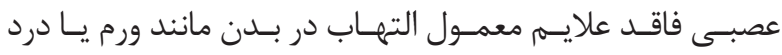

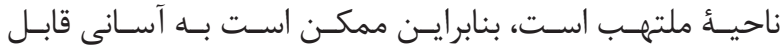

${ }^{44}$ Pervasive developmental disorder

${ }^{45}$ Asperger syndrome

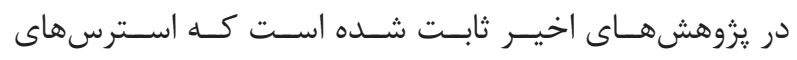

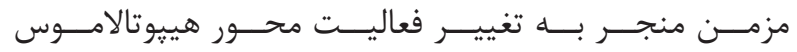

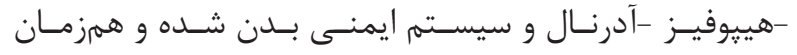

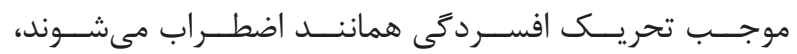

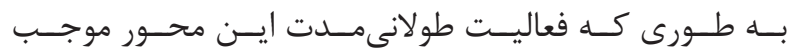

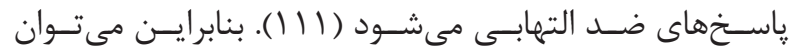

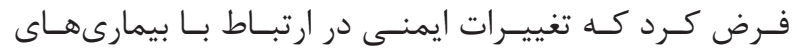

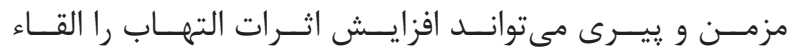

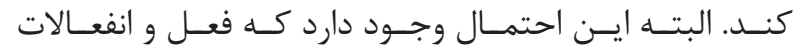

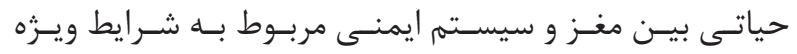

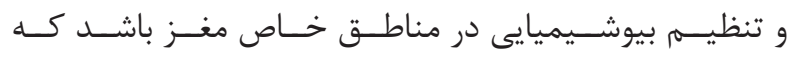

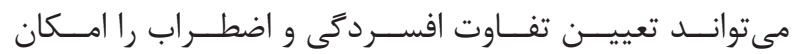

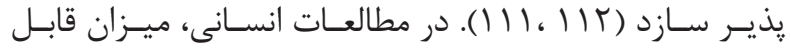

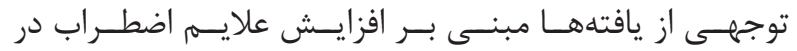

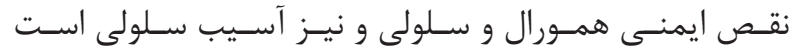

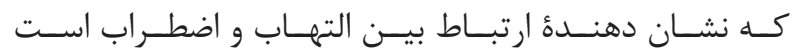

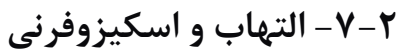

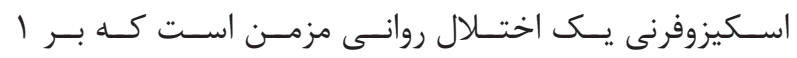

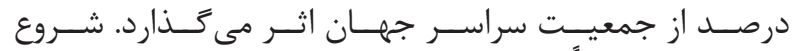

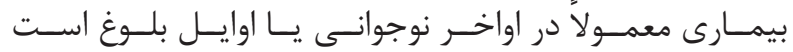

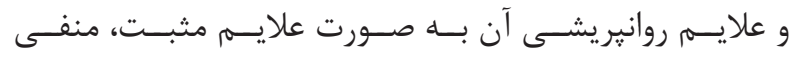

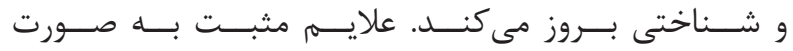

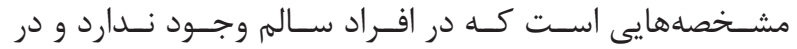

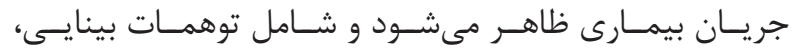

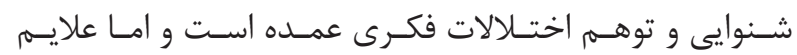

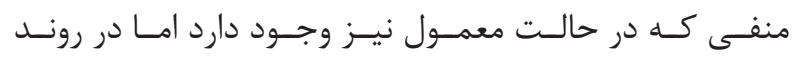

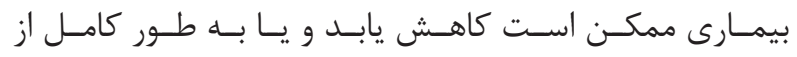

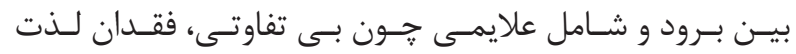

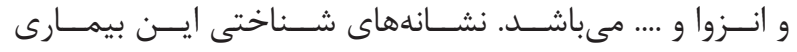

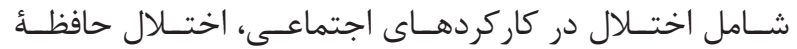

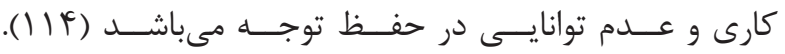

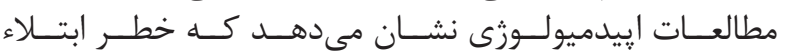

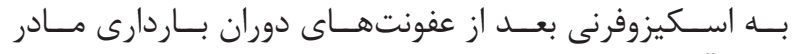

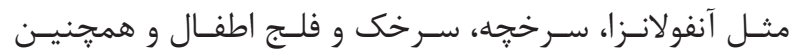

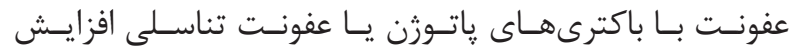

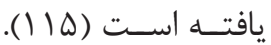

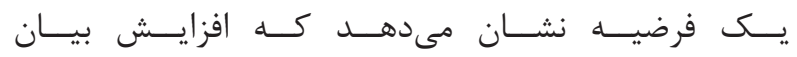

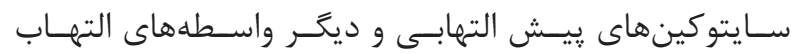

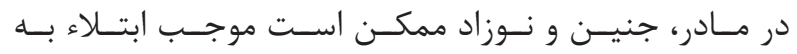

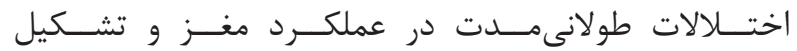

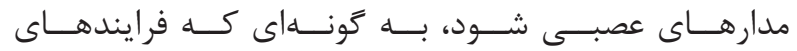

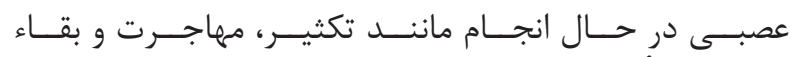

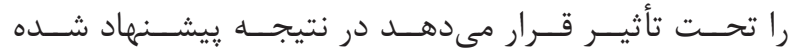

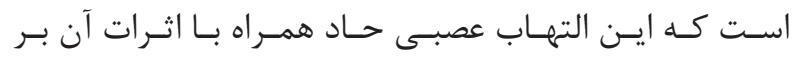

${ }^{46}$ Childhood disintegrative disorder

${ }^{47}$ Synaptogenesis 


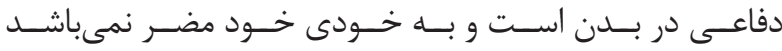

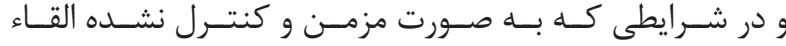

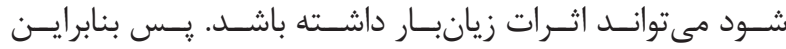

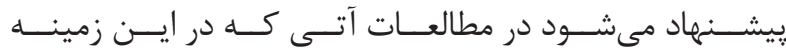

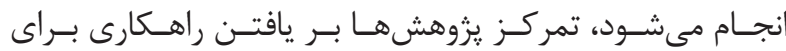

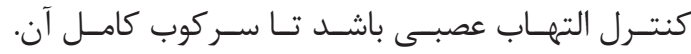

1. Saavedra JM. Angiotensin II AT1 receptor blockers as treatments for inflammatory brain disorders. Clinical Science. 2012; 123(10): 567-90.

2. Hosseini M, Zakeri S, Khoshdast S, Yousefian FT, Rastegar M, Vafaee F, et al. The effects of Nigella sativa hydro-alcoholic extract and thymoquinone on lipopolysaccharide - induced depression like behavior in rats. Journal of Pharmacy \& Bioallied Sciences. 2012; 4(3): 219-25.

3. Pourganji M, Hosseini M, Soukhtanloo M, Zabihi H, Hadjzadeh MA. Protective role of endogenous ovarian hormones against learning and memory impairments and brain tissues oxidative damage induced by lipopolysaccharide. Iranian Red Crescent Medical Journal. 2014; 16(3): e13954.

4. Joshi R, Garabadu D, Teja GR, Krishnamurthy S. Silibinin ameliorates LPS-induced memory deficits in experimental animals. Neurobiol Learn Mem. 2014; 116: 117-31.

5. Saavedra JM. Angiotensin II AT(1) receptor blockers ameliorate inflammatory stress: a beneficial effect for the treatment of brain disorders. Cell Mol Neurobiol. 2012; 32(5): 667-81.

6. Dantzer R, O'Connor JC, Freund GG, Johnson RW, Kelley KW. From inflammation to sickness and depression: when the immune system subjugates the brain. Nat Rev Neurosci. 2008; 9(1): 46-56.

7. Wang X,Zhu M, Hjorth E, Cortés-Toro V, Eyjolfsdottir $\mathrm{H}$, Graff C, et al. Resolution of inflammation is altered in Alzheimer's disease. Alzheimer's \& Dementia. 2015; 11(1): 40-50.

8. Tian Y, Zhang Y, Zhang R, Qiao S, Fan J. Resolvin D2 recovers neural injury by suppressing inflammatory mediators expression in lipopolysaccharide-induced Parkinson's disease rat model. Biochem Biophys Res Commun. 2015; 460(3): 799-805.

9. O’Brien SM, Scully P, Scott LV, Dinan TG. Cytokine profiles in bipolar affective disorder: focus on acutely ill patients. J Affect Disord. 2006; 90(2-3): 263-7.

$$
\begin{aligned}
& \text { تشـخيص نباشــد و بــه مــرور زمـان موجب القـاى اثـرات مخرب }
\end{aligned}
$$

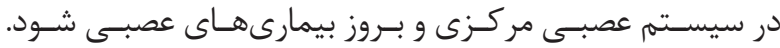

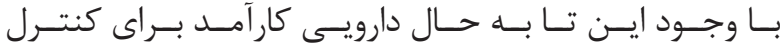

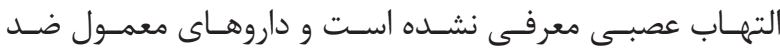

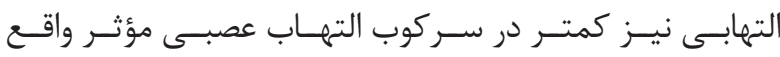

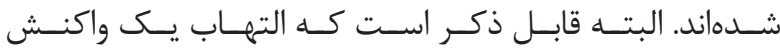

$$
\begin{aligned}
& \text { منابع }
\end{aligned}
$$

10. Müller N, Riedel M, Scheppach C, Brandstätter B, Sokullu S, Krampe K, et al. Beneficial antipsychotic effects of celecoxib add-on therapy compared to risperidone alone in schizophrenia. Am J Psychiatry. 2002; 159(6): 1029-34.

11. Pitsavos C, Panagiotakos DB, Papageorgiou C, Tsetsekou E, Soldatos C, Stefanadis C. Anxiety in relation to inflammation and coagulation markers, among healthy adults: the ATTICA study. Atherosclerosis. 2006; 185(2): 320-6.

12. Vezzani A, Dingledine R, Rossetti AO. Immunity and inflammation in status epilepticus and its sequelae: possibilities for therapeutic application. Expert Rev Neurother. 2015; 15(9): 1081-92.

13. Pascual $\mathrm{M}$, Baliño $\mathrm{P}$, Aragón $\mathrm{CM}$, Guerri $\mathrm{C}$. Cytokines and chemokines as biomarkers of ethanolinduced neuroinflammation and anxiety-related behavior: role of TLR4 and TLR2. Neuropharmacology. 2015; 89: 352-9.

14. Gorelick PB. Role of inflammation in cognitive impairment: results of observational epidemiological studies and clinical trials. Ann N Y Acad Sci. 2010; 1207(1): 155-62.

15. Arakawa H, Blandino P, Deak T. Central infusion of interleukin-1 receptor antagonist blocks the reduction in social behavior produced by prior stressor exposure. Physiol Behav. 2009; 98(1): 139-46.

16. Wyss-Coray $\mathrm{T}$, Mucke $\mathrm{L}$. Inflammation in neurodegenerative disease--a double-edged sword. Neuron. 2002; 35(3): 419-32.

17. Ferrari CC, Tarelli R. Parkinson's disease and systemic inflammation. Parkinson's Disease. 2011; 2011:doi: 436813 .

18. Gonzalez H, Elgueta D, Montoya A, Pacheco R. Neuroimmune regulation of microglial activity involved in neuroinflammation and neurodegenerative diseases. J Neuroimmunol. 2014; 274(1-2): 1-13.

19. Nakagawa Y, Chiba K. Role of microglial M1/M2 


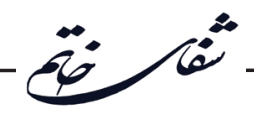

polarization in relapse and remission of psychiatric disorders and diseases. Pharmaceuticals. 2014; 7(12): 1028-48.

20. Prinz M, Priller J. Microglia and brain macrophages in the molecular age: from origin to neuropsychiatric disease. Nature Reviews Neuroscience. 2014; 15(5): 300-12.

21. Cherry JD, Olschowka JA, O'Banion MK. Neuroinflammation and M2 microglia: the good, the bad, and the inflamed. J Neuroinflammation. 2014; 11: 98.

22. Van Gool WA, Van de Beek D, Eikelenboom P. Systemic infection and delirium: when cytokines and acetylcholine collide. The Lancet. 2010; 375(9716): 773-5.

23. Cunningham C, Campion S, Lunnon K, Murray CL, Woods JF, Deacon RM, et al. Systemic inflammation induces acute behavioral and cognitive changes and accelerates neurodegenerative disease. Biol Psychiatry. 2009; 65(4): 304-12.

24. Sankowski R, Mader S, Valdes-Ferrer SI. Systemic inflammation and the brain: novel roles of genetic, molecular, and environmental cues as drivers of neurodegeneration. Front Cell Neurosci. 2015; 9: 28.

25. Holmes C. Review: systemic inflammation and Alzheimer's disease. Neuropathol Appl Neurobiol. 2013; 39(1): 51-68.

26. Cunningham C. Microglia and neurodegeneration: the role of systemic inflammation. Glia. 2013; 61(1): 71-90.

27. Anaeigoudari A, Soukhtanloo M, Shafei MN, Sadeghnia HR, Reisi P, Beheshti F, et al. Neuronal nitric oxide synthase has a role in the detrimental effects of lipopolysaccharide on spatial memory and synaptic plasticity in rats. Pharmacological Reports. 2016; 68(2): 243-9.

28. Anaeigoudari A, Shafei MN, Soukhtanloo M, Sadeghnia HR, Reisi P, Nosratabadi R, et al. The effects of L-arginine on spatial memory and synaptic plasticity impairments induced by lipopolysaccharide. Adv Biomed Res. 2015; 4: 202. doi: 10.4103/22779175.166138 .

29. Azizi-Malekabadi H, Hosseini M, Pourganji M, Zabihi H, Saeedjalali M, Anaeigoudari A. Deletion of ovarian hormones induces a sickness behavior in rats comparable to the effect of lipopolysaccharide. Neurology Research International. 2015; 2015: 627642. doi.org/10.1155/2015/627642.

30. Benicky J, Sánchez-Lemus E, Pavel J, Saavedra
JM. Anti-inflammatory effects of angiotensin receptor blockers in the brain and the periphery. Cell Mol Neurobiol. 2009; 29(6-7): 781-92.

31. Fischer CW, Elfving B, Lund S, Wegener G. Behavioral and systemic consequences of long-term inflammatory challenge. J Neuroimmunol. 2015; 288: 40-6.

32. Roth J, Harre EM, Rummel C, Gerstberger $\mathrm{R}$, Hubschle T. Signaling the brain in systemic inflammation: role of sensory circumventricular organs. Front Biosci. 2004; 9: 290-300.

33. SaavedraJM, Sánchez-LemusE, BenickyJ.Blockade of brain angiotensin II AT 1 receptors ameliorates stress, anxiety, brain inflammation and ischemia: therapeutic implications. Psychoneuroendocrinology. 2011; 36(1): 1-18.

34. Benicky J, Sánchez-Lemus E, Honda M, Pang $\mathrm{T}$, Orecna M, Wang J, et al. Angiotensin II AT1 receptor blockade ameliorates brain inflammation. Neuropsychopharmacology. 2011; 36(4): 857-70.

35. Verma S, Nakaoke R, Dohgu S, Banks WA. Release of cytokines by brain endothelial cells: a polarized response to lipopolysaccharide. Brain Behav Immun. 2006; 20(5): 449-55.

36. Sanchez-Lemus E, Murakami Y, LarrayozRoldan IM, Moughamian AJ, Pavel J, Nishioku T, et al. Angiotensin II AT1 receptor blockade decreases lipopolysaccharide-induced inflammation in the rat adrenal gland. Endocrinology. 2008; 149(10): 5177-88.

37. Banks WA, Robinson SM. Minimal penetration of lipopolysaccharide across the murine blood-brain barrier. Brain Behav Immun. 2010; 24(1): 102-9.

38. Bazyar Y, Rafiei S, Hosseini A, Edalatmanesh MA. Effect of endurance exercise training and gallic acid on tumor necrosis factor- $\alpha$ in an animal model of Alzheimer's disease. Shefaye Khatam. 2015; 3(3): 21-6.

39. Association As. 2016 Alzheimer's disease facts and figures. Alzheimer's \& Dementia. 2016; 12(4): 459509.

40. Werner P, Savva GM, Maidment I, Thyrian JR, Fox C. Dementia: introduction, epidemiology and economic impact. Mental Health and Older People: Springer; 2016. p. 197-209.

41. Higuchi M, Ji B, Maeda J, Sahara N, Suhara T. In vivo imaging of neuroinflammation in Alzheimer's disease. Clin Exp Neuroimmunol. 2016; 7(2): 139-44.

42. Mufson EJ, Ikonomovic MD, Counts SE, Perez SE, 
Malek-Ahmadi M, Scheff SW, et al. Molecular and cellular pathophysiology of preclinical Alzheimer's disease. Behavioural Brain Research. 2016; 311: 54-69.

43. Masters CL, Simms G, Weinman NA, Multhaup G, McDonald BL, Beyreuther K. Amyloid plaque core protein in Alzheimer disease and Down syndrome. Proc Natl Acad Sci USA. 1985; 82(12): 4245-9.

44. Reed-Geaghan EG, Savage JC, Hise AG, Landreth GE. CD14 and toll-like receptors 2 and 4 are required for fibrillar $A \beta$-stimulated microglial activation. The Journal of Neuroscience. 2009; 29(38): 11982-92.

45. Hyman B. Tau and alzheimer pathobiology. Neurobiol Aging. 2016; 39(1): S16.

46. Schliebs R, Arendt T. The significance of the cholinergic system in the brain during aging and in Alzheimer's disease. Journal of Neural Transmission. 2006; 113(11): 1625-44.

47. Trollor JN, Smith E, Agars E, Kuan SA, Baune BT, Campbell L, et al. The association between systemic inflammation and cognitive performance in the elderly: the sydney memory and ageing study. Age (Dordr). 2012; 34(5): 1295-308.

48. Jin DQ, Sung JY, Hwang YK, Kwon KJ, Han $\mathrm{SH}$, Min SS, et al. Dexibuprofen $(\mathrm{S}(+)$-isomer ibuprofen) reduces microglial activation and impairments of spatial working memory induced by chronic lipopolysaccharide infusion. Pharmacology, Biochemistry, and Behavior. 2008; 89(3): 404-11.

49. Kotilinek LA, Westerman MA, Wang Q, Panizzon K, Lim GP, Simonyi A, et al. Cyclooxygenase-2 inhibition improves amyloid-beta-mediated suppression of memory and synaptic plasticity. Brain. 2008; 131(Pt 3): 651-64.

50. Tuppo EE, Arias HR. The role of inflammation in Alzheimer's disease. Int J Biochem Cell Biol. 2005; 37(2): 289-305.

51. de Lau LM, Breteler MM. Epidemiology of Parkinson's disease. The Lancet Neurology. 2006; 5(6): 525-35.

52. Ahmadi M, Sharifi MS. Treatments of Parkinson's disease, epilepsy and obsessive compulsive disorder with deep brain stimulation. Shefaye Khatam. 2014; 2(1): 95-100.

53. Liu B, Jiang J-W, Wilson BC, Du L, Yang S-N, Wang J-Y, et al. Systemic infusion of naloxone reduces degeneration of rat substantia nigral dopaminergic neurons induced by intranigral injection of lipopolysaccharide. J Pharmacol Exp Ther. 2000;
295(1): $125-32$.

54. Levy OA, Malagelada C, Greene LA. Cell death pathways in Parkinson's disease: proximal triggers, distal effectors, and final steps. Apoptosis. 2009; 14(4): 478-500.

55. Vila M, Jackson-Lewis V, Guégan C, Teismann $\mathrm{P}$, Choi D-K, Tieu K, et al. The role of glial cells in Parkinson's disease. Curr Opin Neurol. 2001; 14(4): 483-9.

56. Liu B, Hong J-S. Role of microglia in inflammationmediated neurodegenerative diseases: mechanisms and strategies for therapeutic intervention. J Pharmacol Exp Ther. 2003; 304(1): 1-7.

57. Kreutzberg GW. Microglia: a sensor for pathological events in the CNS. Trends Neurosci. 1996; 19(8): 312-8.

58. Collins LM, Toulouse A, Connor TJ, Nolan YM. Contributions of central and systemic inflammation to the pathophysiology of Parkinson's disease. Neuropharmacology. 2012; 62(7): 2154-68.

59. Ali I, Chugh D, Ekdahl CT. Role of fractalkineCX3CR1 pathway in seizure-induced microglial activation, neurodegeneration, and neuroblast production in the adult rat brain. Neurobiol Dis. 2015; 74: 194-203.

60. Zychowska M, Rojewska E, Makuch W, Przewlocka B, Mika J. The influence of microglia activation on the efficacy of amitriptyline, doxepin, milnacipran, venlafaxine and fluoxetine in a rat model of neuropathic pain. Eur J Pharmacol. 2015; 749: 115-23.

61. Kurkowska-Jastrzebska I, Babiuch M, Joniec I, Przybylkowski A, Czlonkowski A, Czlonkowska A. Indomethacin protects against neurodegeneration caused by MPTP intoxication in mice. Int Immunopharmacol. 2002; 2(8): 1213-8.

62. Sanchez-Pernaute R, Ferree A, Cooper O, Yu $\mathrm{M}$, Brownell AL, Isacson O. Selective COX-2 inhibition prevents progressive dopamine neuron degeneration in a rat model of Parkinson's disease. J Neuroinflammation. 2004; 1(1): 6. doi: 10.1186/17422094-1-6.

63. Teismann P, Tieu K, Choi DK, Wu DC, Naini A, Hunot $\mathrm{S}$, et al. Cyclooxygenase-2 is instrumental in Parkinson's disease neurodegeneration. Proceedings of the National Academy of Sciences of the United States of America. 2003; 100(9): 5473-8.

64. Pott Godoy MC, Tarelli R, Ferrari CC, Sarchi MI, Pitossi FJ. Central and systemic IL-1 exacerbates 


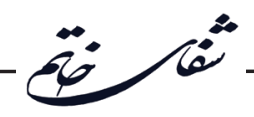

neurodegeneration and motor symptoms in a model of Parkinson's disease. Brain. 2008; 131(7): 1880-94.

65. MacDonald ME, Ambrose CM, Duyao MP, Myers RH, Lin C, Srinidhi L, et al. A novel gene containing a trinucleotide repeat that is expanded and unstable on Huntington's disease chromosomes. Cell. 1993; 72(6): 971-83.

66. Chang K-H, Wu Y-R, Chen Y-C, Chen C-M. Plasma inflammatory biomarkers for Huntington's disease patients and mouse model. Brain, Behavior, and Immunity. 2015; 44: 121-7.

67. Patassini S, Begley P, Xu J, Church SJ, Reid SJ, $\mathrm{Kim} \mathrm{EH}$, et al. Metabolite mapping reveals severe widespread perturbation of multiple metabolic processes in Huntington's disease human brain. Biochimica et Biophysica Acta (BBA) - Molecular Basis of Disease. 2016; 1862(9): 1650-62.

68. Budworth H, McMurray CT. Problems and solutions for the analysis of somatic CAG repeat expansion and their relationship to Huntington's disease toxicity. Rare Diseases. 2016; 4(1): e1131885. doi: $\quad 10.1080 / 21675511.2015 .1131885$.

69. Valera A, Diaz-Hernandez M, Hernandez F, Ortega Z, Lucas J. The ubiquitin-proteasome system in Huntington's disease. The Neuroscientist. 2005; 11(6): 583-94.

70. Cha J-HJ. Transcriptional signatures in Huntington's disease. Prog Neurobiol. 2007; 83(4): 228-48.

71. Stack EC, Matson WR, Ferrante RJ. Evidence of oxidant damage in Huntington's disease: translational strategies using antioxidants. Ann N Y Acad Sci. 2008; 1147(1): 79-92.

72. Browne SE. Mitochondria and Huntington's disease pathogenesis. Ann N Y Acad Sci. 2008; 1147(1): 358-82.

73. Hsiao H-Y, Chiu F-L, Chen C-M, Wu Y-R, Chen $\mathrm{H}-\mathrm{M}$, Chen Y-C, et al. Inhibition of soluble tumor necrosis factor is therapeutic in Huntington's disease. Hum Mol Genet. 2014; 23(16): 4328-44.

74. Clark LF, Kodadek T. The immune system and neuroinflammation as potential sources of bloodbased biomarkers for Alzheimer's disease, Parkinson's disease, and Huntington's disease. ACS Chemical Neuroscience. 2016; 7(5): 520-7.

75. Crotti A, Glass CK. The choreography of neuroinflammation in Huntington's disease. Trends Immunol. 2015; 36(6): 364-73.

76. Hsiao HY, Chern Y. Targeting glial cells to elucidate the pathogenesis of Huntington's disease. Mol Neurobiol. 2010; 41(2-3): 248-55.

77. Regenhardt RW, Desland F, Mecca AP, Pioquinto DJ, Afzal A, Mocco J, et al. Anti-inflammatory effects of angiotensin-(1-7) in ischemic stroke. Neuropharmacology. 2013; 71: 154-63.

78. Andre R, Carty L, Tabrizi SJ. Disruption of immune cell function by mutant huntingtin in Huntington's disease pathogenesis. Current Opinion in Pharmacology. 2016; 26: 33-8.

79. Björkqvist M, Wild EJ, Thiele J, Silvestroni A, Andre R, Lahiri N, et al. A novel pathogenic pathway of immune activation detectable before clinical onset in Huntington's disease. J Exp Med. 2008; 205(8): 1869-77.

80. Nambron R, Silajdzic E, Kalliolia E, Ottolenghi C, Hindmarsh P, Hill NR, et al. A metabolic study of huntington's disease. PloS One. 2016; 11(1): e0146480. doi.org/10.1371/journal.pone.0146480.

81. Kakuma R, Minas H, van Ginneken N, Dal Poz MR, Desiraju K, Morris JE, et al. Human resources for mental health care: current situation and strategies for action. The Lancet. 2011; 378(9803): 1654-63.

82. Furtado M, Katzman MA. Examining the role of neuroinflammation in major depression. Psychiatry Res. 2015; 229(1-2): 27-36.

83. Ferrari AJ, Charlson FJ, Norman RE, Patten SB, Freedman G, Murray CJ, et al. Burden of depressive disorders by country, sex, age, and year: findings from the global burden of disease study 2010. PLoS Medicine. 2013; 10(11): e1001547. doi.org/10.1371/ journal.pmed.1001547.

84. Kessler RC, Petukhova M, Sampson NA, Zaslavsky AM, Wittchen HU. Twelve-month and lifetime prevalence and lifetime morbid risk of anxiety and mood disorders in the United States. Int J Methods Psychiatr Res. 2012; 21(3): 169-84.

85. Wuwongse S, Chang RC-C, Law AC. The putative neurodegenerative links between depression and Alzheimer's disease. Prog Neurobiol. 2010; 91(4): 362-75.

86. Raison CL, Capuron L, Miller AH. Cytokines sing the blues: inflammation and the pathogenesis of depression. Trends Immunol. 2006; 27(1): 24-31.

87. Miller AH, Maletic V, Raison CL. Inflammation and its discontents: the role of cytokines in the pathophysiology of major depression. Biol Psychiatry. 2009; 65(9): 732-41. 
88. McKay MS, Zakzanis KK. The impact of treatment on HPA axis activity in unipolar major depression. J Psychiatr Res. 2010; 44(3): 183-92.

89. Pavón L, Sandoval-López G, Hernández ME, Loría F, Estrada I, Pérez M, et al. Th2 cytokine response in major depressive disorder patients before treatment. J Neuroimmunol. 2006; 172(1): 156-65.

90. Dowlati Y, Herrmann N, Swardfager W, Liu H, Sham L, Reim EK, et al. A meta-analysis of cytokines in major depression. Biological Psychiatry. 2010; 67(5): 446-57.

91. Zellweger MJ, Osterwalder RH, Langewitz W, Pfisterer ME. Coronary artery disease and depression. Eur Heart J. 2004; 25(1): 3-9.

92. Margaretten M, Julian L, Katz P, Yelin E. Depression in patients with rheumatoid arthritis: description, causes and mechanisms. Int J Clin Rheumtol. 2011; 6(6): 617-23.

93. Bonaccorso S, Marino V, Biondi M, Grimaldi F, Ippoliti F, Maes M. Depression induced by treatment with interferon-alpha in patients affected by hepatitis C virus. J Affect Disord. 2002; 72(3): 237-41.

94. Suarez EC, Krishnan RR, Lewis JG. The relation of severity of depressive symptoms to monocyteassociated proinflammatory cytokines and chemokines in apparently healthy men. Psychosom Med. 2003; 65(3): 362-8.

95. Na K-S, Lee KJ, Lee JS, Cho YS, Jung H-Y. Efficacy of adjunctive celecoxib treatment for patients with major depressive disorder: a metaanalysis. Progress in Neuro-Psychopharmacology and Biological Psychiatry. 2014; 48(3): 79-85.

96. Amhaoul H, Staelens S, Dedeurwaerdere S. Imaging brain inflammation in epilepsy. Neuroscience. 2014; 279: 238-52.

97. Sajadian A, Jafarian M, Khodaie B, Mohammad Sadeghi S, Ghaemi A. Reduction of neuroinflammation in Epilepsy by using induced pluripotent stem (ips) cells-derived astrocytes. Shefaye Khatam. 2014; 2(2): 56-64.

98. Vezzani A, Balosso S, Ravizza T. The role of cytokines in the pathophysiology of Epilepsy. Brain, Behavior, and Immunity. 2008; 22(6): 797-803.

99. Vezzani A. Epilepsy and inflammation in the brain: overview and pathophysiology. Epilepsy Currents. 2014; 14(2): 3-7.

100. Macdonald RL, Rogawski MA. Cellular effects of antiepileptic drugs in Epilepsy: a comprehensive textbook. $2^{\text {nd }}$ ed. Wolters Kluwer/Lippincott Williams \& Wilkins. 2008. pp. 1433-45.

101. Gao H-M, Liu B, Zhang W, Hong J-S. Novel antiinflammatory therapy for Parkinson's disease. Trends Pharmacol Sci. 2003; 24(8): 395-401.

102. Vezzani A, Maroso M, Balosso S, Sanchez M-A, Bartfai T. IL-1 receptor/toll-like receptor signaling in infection, inflammation, stress and neurodegeneration couples hyperexcitability and seizures. Brain, Behavior, and Immunity. 2011; 25(7): 1281-9.

103. Rantala H, Tarkka R, Uhari M. Systematic review of the role of prostaglandins and their synthetase inhibitors with respect to febrile seizures. Epilepsy Research. 2001; 46(3): 251-7.

104. Vezzani A, Aronica E, Mazarati A, Pittman QJ. Epilepsy and brain inflammation. Exp Neurol. 2013; 244: 11-21.

105. Kessler RC, Chiu WT, Demler O, Walters EE. Prevalence, severity, and comorbidity of 12-month DSM-IV disorders in the national comorbidity survey replication. Arch Gen Psychiatry. 2005; 62(6): 617-27.

106. Seldenrijk A, Vogelzangs N, van Hout HP, van Marwijk HW, Diamant M, Penninx BW. Depressive and anxiety disorders and risk of subclinical atherosclerosis: findings from the Netherlands study of depression and anxiety (NESDA). J Psychosom Res. 2010; 69(2): 203-10.

107. Carroll D, Phillips AC, Thomas GN, Gale CR, Deary I, Batty GD. Generalized anxiety disorder is associated with metabolic syndrome in the Vietnam experience study. Biol Psychiatry. 2009; 66(1): 91-3.

108. Roest AM, Martens EJ, de Jonge P, Denollet J. Anxiety and risk of incident coronary heart disease: a meta-analysis. J Am Coll Cardiol. 2010; 56(1): 38-46.

109. Huffman JC, Celano CM, Januzzi JL. The relationship between depression, anxiety, and cardiovascular outcomes in patients with acute coronary syndromes. Neuropsychiatr Dis Treat. 2010; 6(6): 123-36.

110. Liukkonen T, Räsänen $P$, Jokelainen J, Leinonen M, Järvelin M-R, Meyer-Rochow V, et al. The association between anxiety and C-reactive protein (CRP) levels: results from the northern finland 1966 birth cohort study. Eur Psychiatry. 2011; 26(6): 363-9.

111. Miller GE, Cohen S, Ritchey AK. Chronic psychological stress and the regulation of proinflammatory cytokines: a glucocorticoid-resistance 


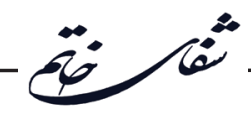

model. Health Psychology. 2002; 21(6): 531-41.

112. Wirtz PH, Von KÄnel R, Schnorpfeil P, Ehlert U, Frey K, Fischer JE. Reduced glucocorticoid sensitivity of monocyte interleukin- 6 production in male industrial employees who are vitally exhausted. Psychosomatic Med. 2003; 65(4): 672-8.

113. Salim S, Chugh G, Asghar M. Inflammation in anxiety. Adv Protein Chem Struct Biol. 2012; 88: 1-25.

114. Tandon R, Nasrallah HA, Keshavan MS. Schizophrenia, "just the facts" 4. clinical features and conceptualization. Schizophr Res. 2009; 110(1): 1-23.

115. Brown AS, Derkits EJ. Prenatal infection and schizophrenia: a review of epidemiologic and translational studies. The American Journal of Psychiatry. 2010; 167(3): 261-80.

116. Gilmore JH, Jarskog LF. Exposure to infection and brain development: cytokines in the pathogenesis of schizophrenia. Schizophr Res. 1997; 24(3): 365-7.

117. Brown AS, Hooton J, Schaefer CA, Zhang H, Petkova E, Babulas V, et al. Elevated maternal interleukin-8 levels and risk of schizophrenia in adult offspring. American Journal of Psychiatry. 2004; 161(5): 889-95.

118. Meyer U, Feldon J, Yee BK. A review of the fetal brain cytokine imbalance hypothesis of schizophrenia. Schizophr Bull. 2009; 35(5): 959-72.

119. Loth E, Spooren W, Ham LM, Isaac MB, Auriche-
Benichou C, Banaschewski T, et al. Identification and validation of biomarkers for autism spectrum disorders. Nature Reviews Drug Discovery. 2016; 15(1): 70-3.

120. Lord C, Cook EH, Leventhal BL, Amaral DG. Autism spectrum disorders. Neuron. 2000; 28(2): 355-63.

121. Chez MG, Dowling T, Patel PB, Khanna P, Kominsky M. Elevation of tumor necrosis factoralpha in cerebrospinal fluid of autistic children. Pediatr Neurol. 2007; 36(6): 361-5.

122. Molloy CA, Morrow AL, Meinzen-Derr J, Schleifer K, Dienger K, Manning-Courtney $\mathrm{P}$, et al. Elevated cytokine levels in children with autism spectrum disorder. J Neuroimmunol. 2006; 172(1): 198-205.

123. Ashwood P, Krakowiak P, Hertz-Picciotto I, Hansen R, Pessah I, Van de Water J. Elevated plasma cytokines in autism spectrum disorders provide evidence of immune dysfunction and are associated with impaired behavioral outcome. Brain, Behavior, and Immunity. 2011; 25(1): 40-5.

124. Mostafa GA, Bjørklund G, Urbina MA, AL-Ayadhi LY. The levels of blood mercury and inflammatoryrelated neuropeptides in the serum are correlated in children with autism spectrum disorder. Metab Brain Dis. 2016; 31(3): 593-9.

125. Edmonson CA, Ziats MN, Rennert OM. A noninflammatory role for microglia in Autism spectrum disorders. Front Neurol. 2016; 7: 9. doi: 10.3389/ fneur.2016.00009. 